\title{
Motivating the Journey:
}

\section{An Integrative Framework of Prevention Versus Promotion Goal Pursuit Activities}

\author{
Emily Nakkawita ${ }^{a} \&$ E. Tory Higgins ${ }^{b}$
}

a Department of Psychology, Columbia University, 406 Schermerhorn Hall, 1190 Amsterdam

Ave., New York, NY 10027, United States. Email: ebn2111@ columbia.edu

${ }^{\mathrm{b}}$ Department of Psychology, Columbia University, 406 Schermerhorn Hall, 1190 Amsterdam

Ave., New York, NY 10027, United States. Email: eth1@columbia.edu

\begin{abstract}
Author Note
Emily Nakkawita (D) https://orcid.org/0000-0002-5400-1311

E. Tory Higgins (D) https://orcid.org/0000-0003-4157-8207
\end{abstract}

This research was completed in partial fulfillment of Emily Nakkawita's MA degree requirements at Columbia University. Parts of this research were featured in presentations at Annual Meetings of the Society for the Science of Motivation and the Society for Personality and Social Psychology. This research did not receive any specific grant from funding agencies in the public, commercial, or not-for-profit sectors. We thank the members of the Higgins Lab for their helpful comments on this research. We also thank Niall Bolger for his statistical guidance with respect to our multilevel modeling approach. Finally, we are grateful to our research assistants for their efforts in helping to conduct this research: Deborah Cesarini, Don Lavelle, Jin Hyung (Kate) Lim, Petra Cui San Poon, and Mo Zhou.

Correspondence concerning this article should be addressed to Emily Nakkawita, Department of Psychology, Columbia University, 406 Schermerhorn Hall, 1190 Amsterdam Ave., New York, NY 10027, United States. Email: ebn2111@ columbia.edu 


\begin{abstract}
Does a focus on gains versus non-losses influence the kinds of activities people are motivated to use when pursuing their goals? This paper proposes that the prevention and promotion systems posited within regulatory focus theory motivate fundamentally different activities in the process of goal pursuit. We present a novel, integrative framework of regulatory focus-specific goal pursuit process activities and provide initial evidence testing this framework. First, across two studies involving activity sorting tasks, we predicted that participants would consistently categorize activities from the proposed framework as reflecting the hypothesized regulatory focus, and in making these categorization decisions, would sort regulatory focus-specific process activities from the proposed framework more quickly than more general goal pursuit process activities. Furthermore, in two follow-up studies probing activity accessibility, we hypothesized that motivationally relevant process activities (i.e., those reflecting individual differences in participants' own regulatory focus) would be more accessible as measured by output primacy than process activities that were not motivationally relevant. Across these four studies, using both correlational and experimental methods, we found converging evidence in support of these predictions and our proposed framework. We suggest that this framework provides new insight into the motivational antecedents of distinct goal pursuit activities. Furthermore, it may be useful in generating new hypotheses about how best to motivate effective goal pursuit processes.

Keywords: regulatory focus, prevention, promotion, goal pursuit, self-regulation,
\end{abstract} motivation 


\section{Motivating the Journey:}

\section{An Integrative Framework of Prevention Versus Promotion Goal Pursuit Activities}

Success is a journey, not a destination. The doing is often more important than the outcome.

-Arthur Ashe

What's in a goal? Several years back, Eitam and Higgins (2014) posed this question in response to a new paper suggesting that active goals trigger patterns of cognition and behavior aimed solely at the attainment of desired end-states (Huang \& Bargh, 2014). Although Huang and Bargh's (2014) proposal fit with a longstanding hedonic perspective on motivation (e.g., Bentham, 1823; Hull, 1950), other scholars have pushed back on this outcome-oriented conceptualization of goal pursuit, suggesting that people also find the process of goal pursuit motivating. As noted by the common adage, it's not only about the destination: How and why people engage in the journey matter.

A number of motivation scientists have theorized about the activities that constitute such journeys (e.g., Carver \& Scheier, 1998; Gollwitzer, 1990; Kruglanski et al., 2000). Although the details of these proposals differ, each advances the notion that goal pursuit processes necessarily involve the dynamic interplay of thought and action, planning and doing, looking and leaping. As described by Atkinson (1957, p. 359), “The first problem is to account for an individual's selection of one path of action among a set of possible alternatives. The second problem is to account for the amplitude of the action tendency once it is initiated, and for its tendency to persist for a time in a given situation." Higgins' (2012) theory of motivational effectiveness integrates these perspectives, proposing that people ultimately want to feel effective in three domains. The domain of value relates to the attainment of desired and undesired end-states as described by Huang and Bargh (2014). The two remaining domains — truth and control-relate to feeling 
effective in the process of goal pursuit. Truth motivation captures the desire to feel effective in establishing what is real and right; control motivation involves the desire to feel effective in managing what happens in one's life (Higgins, 2012).

Although there appears to be a fairly broad consensus that the goal pursuit process encompasses both truth- and control-oriented activities, the field's understanding of why people may choose to engage in certain process activities, but not others, remains limited. For instance, with regard to truth, what motivates people to curiously explore novel ideas versus meticulously scrutinize existing ones? With respect to control, why do some people tend to jump quickly into action, whereas others cautiously defend their existing status quo?

We propose that regulatory focus theory (Higgins, 1997, 1998) offers unique insight into these questions. Regulatory focus theory proposes that two basic self-regulatory systems motivate much of human behavior. The prevention system is motivated by fundamental needs for safety and security; as a result, people with a prevention focus are strategically concerned with approaching desirable end-states involving non-losses and avoiding undesirable end-states involving losses. In contrast, the promotion system is motivated by fundamental needs for nurturance and growth; as a result, people with a promotion focus are strategically concerned with approaching desirable end-states involving gains and avoiding undesirable end-states involving non-gains.

Recently, these different kinds of end-states have also been conceptualized as reference points on a number line (Higgins, 2018). The prevention system treats satisfactory " 0 " status quo states (or anything above them) as desirable reference points and "- 1 " states (or anything below them) as undesirable reference points. In contrast, the promotion system treats " +1 " states (or anything above them) as desirable reference points, whereas " 0 " status quo states (or anything below them) are treated as undesirable reference points. Although it is well-established that the 
prevention and promotion systems are motivated by these different kinds of end-states, relatively less is known about how these systems impact goal pursuit processes: How do promotion and prevention affect the journey of goal pursuit, prior to reaching the destination?

Much of what the field knows about prevention- and promotion-specific goal pursuit processes has been revealed through research related to regulatory fit theory (Higgins, 2000). People experience a state of regulatory fit when the strategic means they use in goal pursuit align with and sustain their motivational orientation, and this kind of fit has important consequences for people's choices to engage in certain activities (Higgins et al., 2010), their enjoyment of such fitting activities (Freitas \& Higgins, 2002), and even their performance (Plessner et al., 2009). Although regulatory fit has been studied with respect to different motivational systems, regulatory fit research examining regulatory focus provides useful insight into the kinds of activities within the process of goal pursuit that might be motivated by the prevention versus promotion systems. People with a prevention focus prefer to pursue goals using vigilant, avoidance-related strategies (e.g., pursuing friendship goals by not losing contact with friends and keeping the secrets friends have shared). In contrast, people with a promotion focus prefer to pursue goals using eager, approach-related strategies (e.g., pursuing friendship goals by being loving, attentive, and generous; Higgins et al., 1994). In addition to research grounded in regulatory fit, the broader regulatory focus literature has grown rapidly since the theory's inception, and a number of these papers offer glimpses into the activities motivated by each system. With this said, we argue that the field could benefit from a more integrated approach to identifying and studying promotion and prevention-specific goal pursuit processes. The present research proposes and tests such a framework. 


\section{Hypothesized Framework of Prevention- and Promotion-Specific Goal Pursuit Activities}

We predict that the prevention and promotion systems' general strategic preferences as well as focus-relevant reference points (i.e., " $-1 "$, , “0”, “+1”) will be reflected in the specific kinds of goal pursuit process activities that are motivated by each system. To this end, we propose a novel, integrative framework of prevention and promotion goal pursuit activities in the domains of truth and control. In the sections that follow, we outline the conceptual and empirical support for this proposal.

\section{Activities Associated with Prevention}

Because the prevention system is motivated to avoid "-1" loss states and maintain a satisfactory " 0 " non-loss status quo, we predict that these reference point preferences will also apply to the domains of truth and control. In the domain of truth, a " -1 " reference point would seem to reflect incorrect, mistaken, or uncertain knowledge. Given the prevention system's predilection for vigilance - that is, avoiding matches to undesired end-states (Crowe \& Higgins, 1997; Higgins et al., 1994) — a vigilant truth process would tend to prioritize satisfactory "0" states like certainty, rightness, and accuracy that reflect the resolution of these " -1 " deficits in truth. Thus, we propose that truth-oriented goal pursuit activities motivated by prevention would likely involve the careful, thorough review and scrupulous verification of information. Although relatively little empirical work has tested this proposal, some research has shown that a prevention focus is associated with: accuracy (vs. speed) in decision making (Förster et al., 2003); analytical (vs. creative) problem solving (Friedman \& Förster, 2005); a preference for using deliberative reasoning ( $v$ s. intuitive feelings) when making judgments (Avnet \& Higgins, 2006; Cornwell \& Higgins, 2016); and increased discounting of negative (vs. positive) prospects over uncertainty (Halamish et al., 2008). 
In the domain of control, a " 0 ” satisfactory status quo reference point would seem to involve the active maintenance of an acceptable present situation in one's life, whereas a "- 1 " reference point would reflect the absence or loss of this satisfactory state. Given the prevention system's preference for vigilance, a prevention-focused control process would tend to prioritize preservation, stewardship, and protection. As a result, we propose that control-oriented goal pursuit activities motivated by prevention would likely involve the cautious defense of the present status quo against potential threats. This proposal is supported by work suggesting that prevention-focused leaders will tend to value conservation values like tradition and conformity (Kark \& Van Dijk, 2007) and preserve interpersonal norms (Zhang et al., 2011). Further, a prevention focus is associated with conservative political ideology (Cornwell \& Higgins, 2013) and decision making (Boldero \& Higgins, 2011), both of which involve the perpetuation of the current status quo. Finally, the prevention system tends to prefer stability over change (Liberman et al., 1999).

\section{Activities Associated with Promotion}

We took a similar approach when conceptualizing promotion goal pursuit process activities. Because the promotion system is motivated to approach " +1 " gain states and avoid the " 0 " non-gain status quo, we first considered how these states would apply in the domain of truth. A " $+1 "$ truth-oriented reference point would seem to reflect the generation of new ideas and knowledge, as well as the growth of one's understanding, whereas a " 0 " reference point would capture a state of mental stagnation. Given the promotion system's preference for eagernessthat is, approaching matches to desired end-states (Crowe \& Higgins, 1997; Higgins et al., 1994) — an eager truth process would likely prioritize curiosity, ideation, and discovery. Thus, we propose that truth-oriented goal pursuit process motivated by promotion would tend to involve curious exploration, brainstorming, and openly seeking out new ideas. Some empirical work 
supports this proposal. For instance, a promotion focus is associated with greater experiences of curiosity (Wytykowska \& Gabińska, 2015); divergent thinking (Beuk \& Basadur, 2016;

Memmert et al., 2013); abstract, high-level construals (Lee et al., 2010); creative (vs. analytical) problem solving (Friedman \& Förster, 2005); and innovation (Wallace et al., 2016).

Turning to the domain of control, a " +1 " reference point would seem to involve managing what happens in one's life by efficiently and smoothly effecting change, whereas a " 0 " reference point would reflect the feeling of being stuck in one's status quo. Given the promotion system's preference for eagerness, a promotion-focused control process would tend to prioritize the advancement and acceleration of goal-directed action. As a result, we propose that controloriented goal pursuit activities motivated by promotion would likely involve moving forward quickly in one's goal pursuits and implementing change processes. This proposal is supported by work suggesting that the promotion system is positively associated with the regulatory mode of locomotion, a motivational orientation concerned with movement and change (Higgins et al., 2008; Kruglanski et al., 2000). Furthermore, a promotion focus is known to be associated with speed (vs. accuracy) in decision making (Förster et al., 2003); openness to change and transformational, change-oriented leadership (Kark \& Van Dijk, 2007); and a preference for political reform (Boldero \& Higgins, 2011).

\section{Proposed Framework}

Based on the theory and research reviewed above, we propose the framework of prevention- and promotion-oriented goal pursuit process activities presented in Table 1.

\section{Table 1}

Hypothesized Framework of Prevention versus Promotion Goal Pursuit Process Activities

\begin{tabular}{cc}
\hline Prevention & Promotion \\
\hline $\begin{array}{c}\text { Truth-Oriented Activities } \\
\text { Scrutiny and Verification }\end{array}$ & Knowledge Growth and Generation
\end{tabular}




\section{Prevention Promotion}

\section{Control-Oriented Activities}

Status-Quo Preservation Movement and Change

Note: For complete lists of the specific activities tested in each study, see the Supplementary Material.

In order to test this framework, we will investigate several hypotheses grounded in basic principles of knowledge activation (Higgins, 1996) and motivational relevance (Eitam \& Higgins, 2010). First, to validate the framework's general applicability, we will examine the availability and accessibility of the associations between the prevention versus promotion systems and process-related activities in each domain. Higgins (1996, p. 134) defines availability as "whether or not some particular knowledge is actually stored in memory." If the proposed associations are unavailable to participants, we would not expect to find any consistency in participants' associations between activities in these four domains and the prevention versus promotion systems. Furthermore, accessibility is defined as "the activation potential of available knowledge" (Higgins, 1996, p. 134). If the associations between a given activity and regulatory focus are highly accessible, we expect that they will be put to use quickly. Importantly, accessibility is influenced by a range of factors, including frequent and recent priming. Because the framework comprises everyday goal pursuit activities that are likely to be activated somewhat frequently, if the proposed associations between these activities and regulatory focus are commonly held and fundamental in nature, we expect that participants will endorse them quickly within our studies. As such, we aimed to test two hypotheses:

Hypothesis 1: Participants will consistently categorize goal pursuit process activities from the framework as reflecting the proposed regulatory focus. 
Hypothesis 2: Participants will categorize regulatory focus-specific process activities from the proposed framework more quickly than more general goal pursuit process activities unrelated to the framework.

Additionally, based on Eitam and Higgins' (2010) relevance of a representation (ROAR) framework, we predict that each activity's accessibility to a given participant — and as a result, the degree to which the activity influences the participant's cognition and action-will depend on the motivational relevance of the activity to the participant. Thus, activities associated with the prevention ( $v s$. promotion) system will be more accessible to participants with a predominant prevention focus, and the reverse will be true for promotion. Higgins (1996) describes two distinct measures of accessibility: output primacy, which describes whether a specific construct is recalled first in a given set of responses, and output frequency, which describes how frequently a specific construct is recalled in response to questions. Because memory reflects accessibility driven by motivational relevance (Eitam et al., 2013), the present research operationalizes output primacy as the order in which prevention versus promotion activities are recalled by participants predominant in each regulatory focus. Based on this reasoning, we also aimed to test the following hypothesis:

Hypothesis 3: Participants will output motivationally relevant process activities (i.e., goal pursuit activities that reflect their own predominant regulatory focus) earlier than process activities that are not motivationally relevant.

\section{The Present Research}

To validate the proposed framework and test these hypotheses, we conducted two sets of studies, both of which involved an initial online study followed by a pre-registered replication study conducted in the lab. In Studies $1 \mathrm{~A}$ and 1B, after learning about the key differences between prevention and promotion motivation, participants categorized goal pursuit process 
activities based on this distinction. We tested whether the consistency and speed of participants' categorization decisions reflected the proposed framework of prevention versus promotion goal pursuit activities. Then, in Studies $2 \mathrm{~A}$ and $2 \mathrm{~B}$, we examined how participants' regulatory focus strength — both measured as a chronic individual difference and manipulated experimentallyrelated to the accessibility of motivationally relevant (vs. irrelevant) activities. We report all manipulations, measures, and exclusions in these studies, as well as the method of determining the final sample size.

\section{Exclusion Criteria}

Across all studies, given that our hypotheses relate to the subtle connotations of English words, we originally planned to conduct this research with only native English speakers. While this criterion was communicated within the recruitment materials for the online studies (1A and 2A), we were not permitted to limit recruitment based on this criterion in the lab studies (1B and 2B) given departmental policies. Thus, across all studies, we instituted an exclusion criterion stating that if less than $10 \%$ of any given dataset comprised participants who reported not being native English speakers, then we would exclude all non-native English speaking participants. If $10 \%$ or more of a given dataset comprised participants who reported not being native English speakers, then we would conduct all analyses both including and excluding the non-native English speaker participants, and would report any differences that emerged. (These results did not differ substantially; as such, the results of all analyses conducted among native English speakers are presented in the Supplementary Material.) Additionally, in all studies, we also excluded any participants who did not complete the independent or dependent measures. These exclusion criteria are detailed in the Study 1B and 2B pre-registrations.

In addition to these pre-registered exclusion criteria, based on a recent rise in the field's awareness of fraudulent responses on online survey platforms (see, for example, Kennedy et al., 
2018), we examined all data collected from online samples for responses from participant IP addresses flagged as fraudulent using $\mathrm{R}$ (Version 4.0.2; R Core Team, 2019) and the R package rIP (Version 1.2.0; Kennedy \& Waggoner, 2019). This flag, which is generated by an API administered by IPHub.info, indicates participants' use of proxy and virtual private network (VPN) servers to mask their location. Across the two studies administered to online samples (1A and 2A), we excluded participants with IP addresses flagged as "Recommend Block" by rIP and IPHub.info.

\section{Diversity and Inclusiveness of Sample}

To maximize the diversity and inclusiveness of the present research, each study group consists of two studies conducted among different populations: a first study that was administered on Amazon's Mechanical Turk (“M-Turk”) platform, and a second study that was administered among the student community at a university in the northeastern United States. We chose to sample from these distinct populations in order to enhance the diversity of the studies' participants on different dimensions. In order to achieve greater diversity with respect to participant age, education, and income, we chose to recruit for our initial studies (Studies 1A and 2A) on M-Turk. Then, to complement these studies with replications among a more racially diverse group of participants, we chose to recruit for our replication studies (Studies 1B and 2B) from the university community.

\section{Analytic Strategy}

To account for both fixed and random effects within the analyses, we proceeded with a Bayesian approach to multilevel modeling and parameter estimation using $\mathrm{R}$ (Version 4.0.2; $\mathrm{R}$ Core Team, 2019) and the R package brms (Version 2.13.5; Bürkner, 2017, 2018). Each primary analysis involved a multilevel regression analysis including fixed effects appropriate for the particular study and random effects of both participant and activity. We chose this Bayesian 
approach for several reasons. The first two reasons are conceptual. Unlike frequentist null hypothesis significance testing, Bayesian approaches provide insight into the uncertainty of model estimates and a distribution of credible parameter values (Kruschke, 2011). Furthermore, modeling the goal pursuit activities that we tested as random stimuli reflected our thinking about these activities: Although we do believe that the proposed activity lists reflect the different kinds of goal pursuit processes motivated by prevention versus promotion, we do not claim that these activity lists are complete. For this reason, it is more accurate to treat these activities as a random sample among the larger set of activities associated with prevention versus promotion. The final reason we proceeded with a Bayesian approach was practical, as frequentist models with a complex random effects structure commonly fail to converge, and Bayesian modeling offered a more tractable path forward.

Because the present research reflects the first attempt to study the hypothesized associations between regulatory focus and goal pursuit process activities, we took a conservative approach by proceeding with uninformative prior distributions reflecting no prior knowledge for all variables. All analyses were conducted using four Markov chains. We assessed model convergence by ensuring that all R-hat values fell at or below 1.01, and to ensure each model's success in achieving this convergence benchmark, we used between 2,000 and 4,000 MCMC iterations per chain. In order to draw inferences from these Bayesian multilevel analyses, we followed a common procedure for Bayesian parameter estimation recommended by Kruschke (2011), which involves the examination the $95 \%$ credible intervals around the estimated regression coefficients. If this credible interval excluded 0 , we concluded that the coefficient reflected an effect of interest. The posterior distributions for all effects of interest, including a visualization of these $95 \%$ credible intervals, are provided in the Supplementary Material. Additionally, we only report the fixed effects from these analyses in the main text because, 
although we were interested in accounting for random effects in the models, we did not have any specific hypotheses about them (e.g., with respect to heterogeneity in the effects). However, the complete model output, including both fixed and random effects, are also available in the Supplementary Material.

These models reflect the pre-registered analyses with one exception and two key additions. The exception relates to the inclusion of specific random effects in the models. We are grateful to a colleague who advised that it is recommended not to include any random effect variables that contain less than six levels; as a result, the models do not include random slopes for any variables that fall below this benchmark (e.g., any dummy-coded regulatory focus variables with two levels: prevention $v s$. promotion), which reflects a minor deviation from the preregistered analysis plans. The additions pertain to Studies $2 \mathrm{~A}$ and $2 \mathrm{~B}$, and involve the inclusion of a covariate (a secondary measure of accessibility) and several exploratory analyses that were not pre-registered. More details on these additions, as well as justification for their inclusion, can be found in the Analytic Strategy sections for these studies.

\section{Maximizing Power}

As described in further detail for each study, we sought to maximize statistical power in the present research. First, all studies were designed to manipulate activity regulatory focus as a within-participant factor to enhance the power of the research. Second, we conducted a priori power analyses before conducting each of the present studies; sample size was determined before any data analysis. In Studies 1A and 2A, although our analytic approach is primarily Bayesian, we conducted traditional power analyses using $\mathrm{G}^{*}$ Power (Faul et al., 2009) to determine an appropriate sample size for each of these studies as, given that these lines of inquiry were new, we were unable to confidently proceed with a simulation-based approach. In these studies, we selected a sample size aimed at detecting a medium-sized effect (Cohen, 1988) with power of 
0.80 at $\alpha=0.05$. In Studies $1 \mathrm{~B}$ and $2 \mathrm{~B}$, as pre-registered, we conducted Bayesian equivalents of power analyses following a simulation-based approach recommended by Kurz (2019) using R (Version 4.0.2; R Core Team, 2019). In these analyses, our goal was to obtain power of 0.90 at $\alpha=0.05$ to detect effects of the same size or smaller as those detected in Studies $1 \mathrm{~A}$ and $2 \mathrm{~A}$.

\section{Study 1A}

In Study 1A, after completing two brief questionnaires probing their chronic motivational orientations, participants learned about the distinction between prevention and promotion motivation. Then, participants completed a sorting task in which they categorized verbs as either prevention or promotion activities. This study provided an initial opportunity to test if participants' categorization decisions reflected the proposed framework. Based on Hypothesis 1, we predicted that participants' categorization decisions for goal pursuit process activities that we predicted were associated with either prevention or promotion would consistently reflect the activities' hypothesized regulatory focus in the framework. Further, based on Hypothesis 2, we predicted that participants would categorize regulatory focus-specific process activities from the proposed framework more quickly than more general goal pursuit process activities. Finally, we examined whether participants' categorization decisions were predicted by individual differences in participants' own chronic promotion versus prevention focus. Although subsequent studies (2A, 2B) tested whether participants' regulatory focus influenced the accessibility of relevant activities, in Study 1A, we did not expect that categorization decisions in general (i.e., across all kinds of activities) would vary based upon individual differences in participants' regulatory focus.

\section{Method}

\section{Participants}


We conducted a traditional power analysis aimed at detecting a medium-to-large odds ratio effect of $O R=6$ (Cohen, 1988) with power of 0.80 . This analysis (G*Power; Faul et al., 2009; two-tailed; conditional probability that $Y=1$ given $X=1$ under $H_{0}=0.5 ; \alpha=0.05$; binomial $X$ distribution for which $\pi=0.5$ ) indicated that 56 participants would be required. To account for potential exclusions, we set the target sample size at 75 participants.

Data collection took place in February 2019. Using M-Turk, we recruited 75 native English speaking U.S. workers at least 18 years old. All participants were paid \$2. Two workers provided the correct code for payment without completing the study, leaving a sample of 73 participants. We then examined the dataset for fraudulent responses based on participant IP addresses using the R package $r I P$ (Version 1.2.0; Kennedy \& Waggoner, 2019). Eleven responses were submitted by IP addresses flagged as fraudulent, so these responses were excluded. Finally, we examined the number of participants who disregarded the recruitment criteria and participated despite their alternate native language. Only one participant reported a native language other than English, and because the percentage of non-native English speaking participants $(1.6 \%)$ fell below our $10 \%$ threshold, this participant was excluded.

These exclusions left a sample of 61 participants ranging in age from 24 to $68(M=37$; $S D=12)$. Participants were male $(51 \%)$ and female $(48 \%)$, with the remaining participants unspecified. They self-identified as White (80\%), Black (8\%), Asian (7\%), Hispanic (2\%), and multiracial (2\%). They indicated the highest level of formal education they have completed as a high school diploma (18\%), some college (16\%), an associate degree (10\%), a bachelor's degree (48\%), and a master's degree (8\%). They identified their approximate annual household income as $\$ 0-\$ 10 \mathrm{~K}(5 \%), \$ 10-\$ 20 \mathrm{~K}(7 \%), \$ 20-\$ 40 \mathrm{~K}(21 \%), \$ 40-\$ 70 \mathrm{~K}(31 \%), \$ 70-\$ 100 \mathrm{~K}(18 \%)$, $\$ 100-\$ 250 \mathrm{~K}(13 \%)$, and $\$ 500 \mathrm{~K}+(2 \%)$. A sensitivity analysis (G*Power; Faul et al., 2009) showed that this final sample could detect an effect size of $O R=5.32$ or larger for the effect of 
Hypothesized Activity Regulatory Focus (power=0.80; $\alpha=0.05$; two-tailed; conditional probability that $Y=1$ given $X=1$ under $H_{0}=0.5$; binomial $X$ distribution for which $\pi=0.5$ ).

Finally, as per common practice when working with response latency data (e.g., Carpenter et al., 2019), we excluded activity categorization decisions for which the response latency was too short (under $250 \mathrm{~ms}$ ), thus indicating overly fast responding (i.e., "button mashing"), and too long (over 10,000 ms), indicating a lack of attention. After removing these observations, 6,262 total activity categorization decisions remained, with a mean of 102.66 categorization decisions per participant $(S D=28.54)$.

\section{Materials and Measures}

Preliminary Activity List. To capture the different kinds of goal pursuit process activities associated with prevention versus promotion, we aimed to identify actions that people will take to establish what is real and right (i.e., truth-oriented activities) and manage what happens (i.e., control-oriented activities) when motivated by each system. To generate an initial pool of activities, the authors individually developed lists of verbs for consideration based on extensive conversations about regulatory focus theory and reviews of past empirical work on the unique associations between regulatory focus and variables related to truth and control (see Activities Associated with Prevention and Activities Associated with Promotion sections). We also reviewed several motivational dictionaries that have been developed for use in linguistic analysis, including a regulatory focus dictionary (Gamache et al., 2015) and a regulatory mode dictionary (Kanze et al., 2019), and appended a range of activities that we considered relevant to the proposed framework. We then expanded on these lists using a thesaurus, and refined them through ongoing conceptual discussions. The list of prevention-truth activities included behaviors involving careful evaluation and aimed at certainty, such as scrutinize, judge, and verify. The list of prevention-control activities included behaviors involving the vigilant maintenance and 
protection of a satisfactory status quo, such as defend, preserve, and protect. The list of promotion-truth activities included behaviors involving mental exploration and aimed at knowledge growth and generation, such as imagine, discover, and explore. The list of promotioncontrol activities included behaviors involving the initiation of forward-directed action aimed at effecting change, such as propel, accelerate, and lead.

Additionally, in order to distinguish these prevention- and promotion-specific process activities from a more general group of truth- and control-oriented actions, we also generated a pool of goal pursuit process activities that we hypothesized were not associated with the prevention or promotion systems per se, including general truth activities (e.g., investigate, consider) and general control activities (e.g., manage, operate). Finally, to provide participants with the ability to practice the primary task with stimuli already known to be associated with prevention or promotion, we selected, using Gamache et al.'s (2015) dictionary, six verbs that we believed best reflected the attainment of the desired outcomes of a prevention focus (secure, evade, prevent) and a promotion focus (achieve, gain, grow). Through this procedure, we generated an initial list of 57 activities: 34 promotion- and prevention-specific truth- and controloriented process activities; 17 general truth- and control-oriented process activities; and 6 verbs reflecting the acquisition of promotion- and prevention-specific outcomes. (For a complete list of these activities, see Table S1 in the Supplementary Material.) Importantly, the regulatory focus of each activity hypothesized to relate to regulatory focus was dummy-coded $(0=$ prevention; 1=promotion). Based on these hypotheses, we manipulated activity regulatory focus (i.e., our primary independent variable) on a within-participants basis in the activity sorting task described next.

Activity Sorting Task. Participants categorized each of the verbs in the activity list as prevention or promotion activities within a JavaScript-based sorting task. On-screen instructions 
directed participants to place their left and right index fingers on their E and I keys, respectively. The labels "prevention" and "promotion" were placed at each of the upper corners of the screen. Instructions described that verbs would appear in the middle of the screen, and that participants should indicate the category to which each verb belongs by pressing the associated key. Participants were directed to proceed as fast as they can, while making as few mistakes as possible, in order to respectively balance concerns with speed and accuracy. In all blocks of trials, activities were selected randomly and without replacement until the block's activity list was exhausted. Activities were presented one at a time with an inter-trial interval of 2,000 ms. Each activity remained on-screen until the participant's response, and no feedback was provided after any responses. For each trial, the software recorded the activity, the participant's categorization decision, and the participant's response latency in indicating their decision.

The sorting task in Study 1A comprised two groups of trial blocks. In one group, the "prevention" label was placed in the upper left corner and the "promotion" label was placed in the upper right corner; in the second group, the placement of these labels was reversed. (The placement of these category labels was counterbalanced, such that half of participants were randomly assigned to begin with "prevention" on their left and the other half began with "prevention" on their right.) Each of the two groups consisted of two blocks of trials. The first block was a brief practice round consisting of six brief trials intended to familiarize participants with the logistics of the task, in which participants sorted the six verbs already known to reflect the acquisition of prevention- and promotion-specific outcomes. The second block involved completing 51 additional trials which involved categorizing the 51 process activities of interest as prevention or promotion. Upon completing the second block, the position of the labels was reversed and participants completed the second group of trial blocks. Because participants 
completed the sorting task with the labels in both positions, each participant categorized each activity twice.

Regulatory Focus Pride. To measure chronic historical effectiveness (i.e., "pride”) in the domains of prevention and promotion, we administered the Regulatory Focus Questionnaire (RFQ; Higgins et al., 2001). The RFQ asks participants to respond on five-point scales (1=never or seldom or certainly false to 5=very often or certainly true) to eleven items. Five items measure prevention pride (e.g., "Not being careful enough has gotten me into trouble at times"; $\alpha=0.86$ ) and six items measure promotion pride (e.g., "I feel like I have made progress toward being successful in my life"; $\alpha=0.64)$. After mean endorsement levels were determined for each participant $\left(M_{\text {prevention }}=3.37 ; S D_{\text {prevention }}=0.86 ; M_{\text {promotion }}=3.43 ; S D_{\text {promotion }}=0.61\right)$, we calculated promotion ( $v s$. prevention) pride dominance scores for use in the primary analysis, as these orientations involve competing motivational preferences. As a result, the predominant regulatory focus score reflects a resultant of the two motivational forces, and we were interested in testing if this resultant predicted participants' categorization decisions across all activity types. We calculated these dominance scores by subtracting each participant's prevention pride score from their promotion pride score $(M=0.06 ; S D=1.05)$. Finally, these promotion pride dominance scores were mean-centered for use in the regression analyses so the reference condition for this variable would reflect the typical level of promotion pride dominance among participants. To do so, we subtracted each participant's promotion pride dominance score from the promotion pride dominance grand mean.

Further Measures. To control for any potential effects of stimulus length or frequency of use in the English language, we measured each of these variables for all activities. Activity length was calculated based on the number of characters in each stimulus word. Activity frequency of 
use in the English language was taken from the Corpus of Contemporary American English (Davies, 2008).

For exploratory purposes, we also measured participants' assessment and locomotion orientations using the Regulatory Mode Questionnaire (RMQ; Kruglanski et al., 2000). Assessment and locomotion are self-regulatory functions reflecting specific types of truth and control motivation, respectively. Assessment involves the critical evaluation of goals or means, and locomotion involves effecting change through movement from state to state (Higgins et al., 2003; Kruglanski et al., 2000). The RMQ asks participants to respond on six-point scales (1=strongly disagree to $6=$ strongly agree $)$ to 30 items. Twelve of these items measure locomotion (e.g., "I don't mind doing things even if they involve extra effort"; $M=4.15 ; S D=0.80$; $\alpha=0.86$ ) and twelve others measure assessment (e.g., "I often critique work done by myself and others"; $M=3.61 ; S D=0.80 ; \alpha=0.81$ ). Additionally, we administered four exploratory measures probing participants' experience in completing the activity sorting task. Using a five-point scale (1=extremely easy to 5=extremely difficult), participants indicated: "Overall, how difficult was it to categorize these words as promotion vs. prevention" $(M=2.69 ; S D=1.09)$. Participants also responded to three open-ended questions: "As best you can, please describe how you made your categorization decisions"; "If you remember any words that were especially difficult to categorize, please list them here"; and "Please enter any additional feedback you'd like to provide here". Although responses to these exploratory measures are not discussed further here, they are available within the study dataset provided on the Open Science Framework (OSF) project page associated with the present research (https://osf.io/ybcaq/?view_only=8bf7abc0419f4f618662f6c870aaea60).

\section{Procedure}


After consenting to participate in the study, participants completed two motivational questionnaires: the RFQ and RMQ. Then, participants read that they would learn about two different types of motivation called prevention and promotion. To ensure that participants did not conflate promotion with approach motivation and prevention with avoidance motivation, they were explicitly told that "Both types of motivation want positive, desired outcomes, but the kinds of outcomes on which each type of motivation focuses are different." They learned that prevention is a type of motivation focused on desired outcomes like safety and security, whereas promotion focuses on desired outcomes like achievement and growth. Further, they learned that prevention-focused people prefer to avoid losses, put themselves in situations where they will not lose, and maintain a current satisfactory status quo to avoid worse states. Promotion-focused people prefer to pursue gains, avoid situations where they will not gain, and change the status quo in order to attain a better state. Finally, they learned that people strongly motivated by prevention are focused on the positive things they ought to do in a given situation, so they tend to pursue their goals vigilantly; in contrast, people strongly motivated by promotion are focused on the positive things they would ideally like to do in a given situation, so they tend to pursue their goals eagerly.

After learning these distinctions between prevention and promotion, participants proceeded to the activity sorting task described above. Between each block of trials, the prevention-promotion distinctions first encountered in the initial training were presented again to refresh participants' memories. Finally, after finishing the sorting task, participants completed the exploratory items intended to probe their categorization experience. Participants then optionally provided demographic information and were debriefed, thanked, and compensated.

\section{Results}

\section{Analytic Strategy}


The primary analysis examined whether our predictions about the hypothesized regulatory focus of each prevention or promotion process activity were reflected in participants' categorization decisions. We selected the subset of the data comprising only activities we hypothesized to reflect prevention- or promotion-specific processes $(N=3,724)$ and analyzed these data within a Bayesian multilevel binomial logistic regression model. Participants' categorization decisions (i.e., our primary dependent variable) were dummy-coded for this analysis $(0=$ prevention $; 1=$ promotion $)$, and the same coding scheme was applied to the hypothesized regulatory focus of each activity (i.e., our primary independent variable). The model also included promotion ( $v s$. prevention) pride dominance as a covariate, as we were also interested in testing if categorization decisions were associated with chronic individual differences in regulatory focus. Other covariates included as fixed effects were the initial placement of the category names (effect-coded: $-0.5=r i g h t ;+0.5=l e f t$ ), as well as trial, activity frequency, and activity length (all rescaled: $M=0 ; S D=1$ ). Random effects included in the model were random intercepts of participant and activity; random slopes of trial, activity frequency, and activity length by participant; and random slopes of promotion pride dominance by activity.

The secondary analysis tested whether verbs that we hypothesized were prevention- or promotion-specific process activities were categorized more quickly than general process activities that we hypothesized were not associated with a particular regulatory focus. To conduct this analysis, we analyzed all categorization decisions for the 51 process activities $(N=5,593)$ within a Bayesian multilevel multiple regression model. Participants' response latencies for each categorization decision were log-transformed to normalize the distribution. Then, these logtransformed response latencies were regressed on a dummy-coded variable capturing whether or not each activity was hypothesized to reflect a specific regulatory focus $(0=$ not a prevention or promotion activity; $1=$ prevention or promotion activity). This model included the same covariates 
and random effects as the primary model. (For descriptive statistics and correlations among all variables included within these models, see Table S3 in the Supplementary Material.)

Additionally, we examined the consistency and speed of participants' categorization decisions about each activity in Study 1A in order to refine the activity list. In doing so, we aimed to develop a final pool of 24 activities, with six per category (prevention-truth; preventioncontrol; promotion-truth; promotion-control). We also used the activity length and frequency information to ensure that these four groups did not significantly differ on these dimensions. After making these list refinements, we re-conducted our primary and secondary analyses using this refined activity list, the results of which can be found in the Supplementary Material.

\section{Activity Categorization Decisions}

Among the categorization decisions for all goal pursuit process activities (i.e., both the process activities hypothesized to be regulatory focus-specific as well as general process activities), on average, $47.26 \%$ were categorized as prevention and $52.74 \%$ were categorized as promotion. Among the decisions made with respect to the proposed list of prevention- and promotion-specific goal pursuit process activities, a similar pattern emerged: an average of $47.77 \%$ of activities were categorized as prevention and $52.23 \%$ were categorized as promotion. By estimating the intercept within a Bayesian multilevel logistic regression model including a fixed intercept as well as random intercepts of participant and activity, we determined that the probability of categorizing the hypothesized regulatory focus-specific process activities as promotion ( $\log$ odds of 0.15 units; $95 \%$ CI: $-0.43,0.73$; probability of $53.74 \%$; $95 \%$ CI: $39.33 \%$, $67.43 \%$ ) did not differ significantly from chance (i.e., a probability of 50\%). 
Among all categorization decisions (i.e., for all process activities), on average, activities were categorized in 1.32 seconds, and hypothesized prevention- and promotion-specific goal pursuit process activities were categorized in 1.31 seconds.

\section{Primary Analysis: Do Our Hypotheses About Whether Each Process Activity Reflects a Promotion (vs. Prevention) Focus Predict Participants' Categorization Decisions?}

To test if our hypotheses about the process activities' regulatory focus predicted participants' categorization decisions, we examined the model output from the Bayesian multilevel logistic regression analysis (see the upper panel of Table 2 and Figure 1). The estimated effect of hypothesized activity regulatory focus was $2.82 \log$ odds units $(O R=16.78)$, with a $95 \%$ credible interval that did not include zero $(2.35,3.32)$. This credible interval suggests that there is an association between our hypotheses about the prevention versus promotion focus of goal pursuit process activities and participants' categorization decisions. Transformed into probabilities, the model predicts that activities that we hypothesized were associated with prevention had a $74.25 \%$ probability of being categorized as prevention, and activities that we hypothesized were associated with promotion had an $85.34 \%$ probability of being categorized as promotion.

Additionally, this analysis allowed us to test if participants' categorization of any given process activity as promotion or prevention is a function of the participant's promotion ( $v s$. prevention) pride dominance. As indicated in the upper panel of Table 2, the estimated effect of promotion pride dominance on activity categorization is 0.10 , with a $95 \%$ credible interval that includes zero $(-0.06,0.27)$. This credible interval indicates that we cannot infer any association between individual differences in promotion pride dominance and participants' categorization decisions. Further, no other fixed effects in this primary analysis had a credible interval excluding zero. 


\section{Table 2}

Parameter Estimates for Multilevel Binomial Logistic Regression Analysis Predicting Process Activity Categorization and Multilevel Multiple Regression Analysis Predicting Log-Transformed Response Latency (Study 1A; Fixed Effects Only)

\begin{tabular}{lrrrr}
\hline & & & \multicolumn{2}{c}{$95 \%$ CI } \\
\cline { 4 - 6 } Effect & Estimate & Post.SD & Lower & Upper \\
\hline DV: Activity Categorization (0=Prev.; 1=Prom.) & & & & \\
Intercept & -1.06 & 0.35 & -1.75 & -0.38 \\
Hypothesized Activity RF (0=Prev.; 1=Prom.) & $\mathbf{2 . 8 2}$ & $\mathbf{0 . 2 4}$ & $\mathbf{2 . 3 5}$ & $\mathbf{3 . 3 2}$ \\
Promotion ( $v$. Prevention) Pride Dominance & 0.10 & 0.08 & -0.06 & 0.27 \\
Trial & -0.01 & 0.05 & -0.11 & 0.08 \\
Order of Category Labels & -0.14 & 0.17 & -0.47 & 0.18 \\
Frequency of Use in English Language & 0.38 & 1.62 & -2.79 & 3.60 \\
Length & -0.09 & 0.17 & -0.41 & 0.25 \\
DV: Log-Transformed Response Latency & & & & \\
Intercept & 7.03 & 0.05 & 6.92 & 7.13 \\
Hypothesized RF-Specific Activity (0=No; 1=Yes) & $-\mathbf{0 . 0 5}$ & $\mathbf{0 . 0 2}$ & $-\mathbf{0 . 0 9}$ & $\mathbf{- 0 . 0 1}$ \\
Promotion ( $v$. Prevention) Pride Dominance & -0.01 & 0.04 & -0.09 & 0.08 \\
Trial & -0.03 & 0.01 & -0.05 & -0.01 \\
Order of Category Labels & 0.15 & 0.09 & -0.01 & 0.33 \\
Frequency of Use in English Language & -0.03 & 0.01 & -0.05 & -0.01 \\
Length & 0.01 & 0.01 & -0.01 & 0.03 \\
\hline Note. RF=Regulatory Focus; Prom =Promotion; Prev $=$ Prevention. Effects for the predictor of
\end{tabular}

interest are shown in bold. For the complete results of these analyses, including random effects, see Tables S4 and S5 in the Supplementary Material.

\section{Figure 1}

Probability of Categorization as Promotion (vs. Prevention) as a Function of Hypothesized Activity Regulatory Focus (Study 1A) 


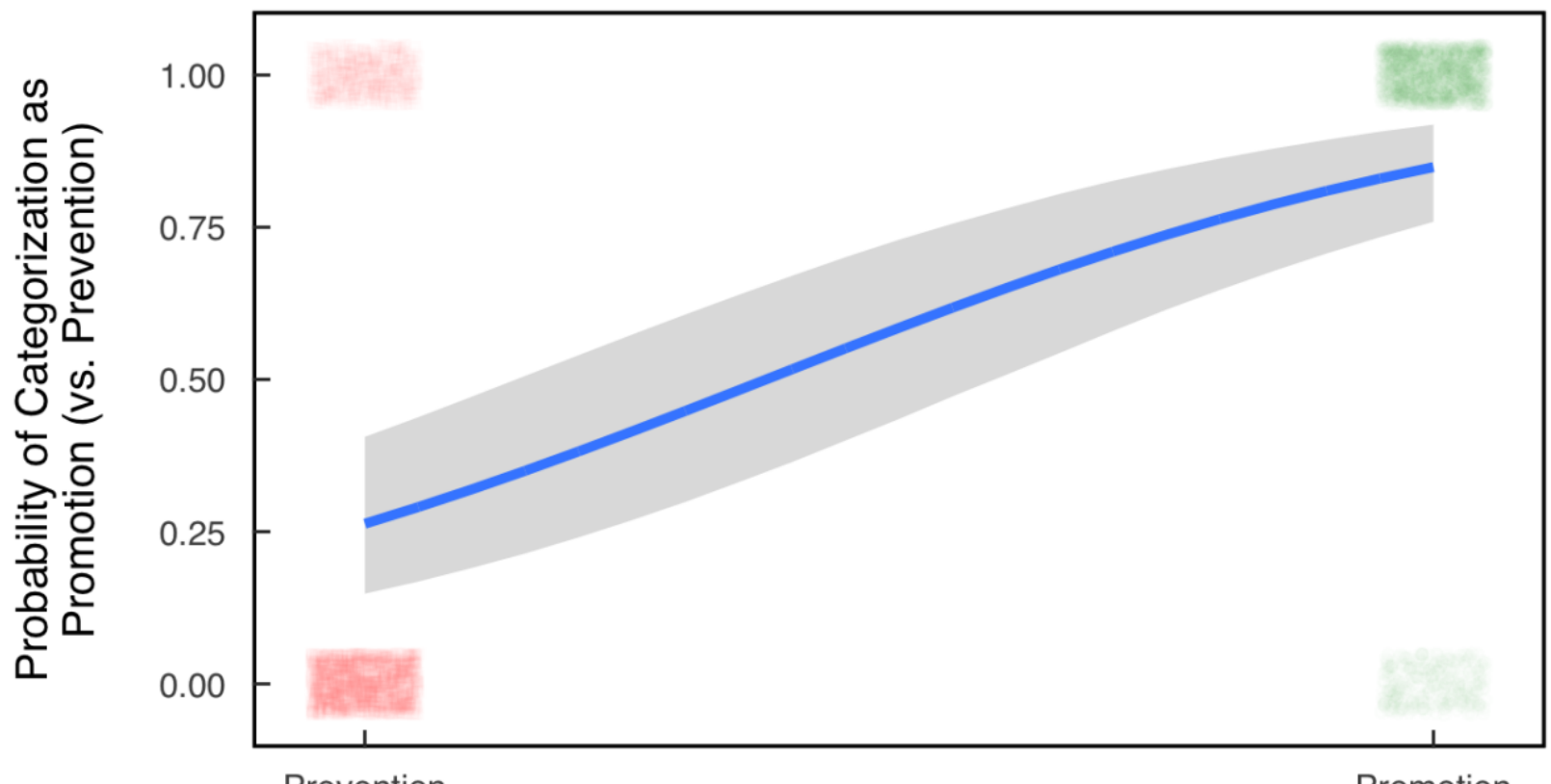

Prevention

Promotion

Hypothesized Regulatory Focus

( $0=$ Prevention; $1=$ Promotion)

$$
\text { Hypothesized Regulatory Focus घ Prevention • Promotion }
$$

Note. Model-predicted probability of categorization as promotion ( $v s$. prevention) as a function of hypothesized activity regulatory focus, at typical levels of promotion pride dominance, trial, order of label presentation, activity frequency of use in the English language, and activity length. The red (dark gray) squares represent categorization decisions for activities hypothesized to reflect prevention processes. The green (light gray) circles represent categorization decisions for activities hypothesized to reflect promotion processes. Shaded region represents $95 \%$ credible interval.

Secondary Analysis: Are Activities Hypothesized to Be Associated with a Specific Regulatory Focus Process Categorized More Quickly Than Process Activities Without These Hypothesized Associations?

To test if our hypotheses about the process activities' associations with regulatory focus (vs. lack thereof) predicted how quickly participants made their categorization decisions, we 
examined the model output from the Bayesian multilevel multiple regression analysis (see the lower panel of Table 2). The estimated effect of this hypothesized association with regulatory focus is -0.05 log-transformed milliseconds, with a $95 \%$ credible interval that did not include zero $(-0.09,-0.01)$. These results suggest that, as hypothesized, participants were faster in categorizing activities that we hypothesized were ( $v s$. were not) associated with a specific regulatory focus process. The model predicts that activities that we hypothesized were associated with a specific regulatory focus process would be categorized in 1.07 seconds, and activities that we hypothesized were not associated with a specific regulatory focus process would be categorized in 1.13 seconds.

Additionally, this analysis allowed us to test if participants' response latencies in categorizing all process activities were associated with their promotion ( $v s$. prevention) pride dominance. As indicated in the lower panel of Table 2, the estimated effect of promotion pride dominance is -0.01 , with a $95 \%$ credible interval that includes zero $(-0.09,0.08)$. These results suggest no association between individual differences in promotion pride dominance and participants' categorization speed. Finally, several covariates were associated with participants' response latency, including trial (such that participants' categorization speed increased over the course of the study) and activities' frequency of use in the English language (such that more frequently used words were categorized more quickly).

\section{Activity List Refinement}

Although the analyses above provided initial support for the proposed framework, given that this study represented our first thorough investigation on this topic, we were interested in refining the activity lists based on these results. We intended to develop a slightly condensed set of process activities with an equal number of activities per category and, in doing so, we ensured that both activity length and frequency of use in the English language did not differ among the 
categories. To accomplish these aims, we engaged in a number of conceptual discussions about the activity categories, informed by a thorough inspection of participants' categorization decisions and response latencies by activity.

Through this process, we determined a final list of 24 prevention- and promotion-specific goal pursuit process activities (see Figure 2 and Table S2 in the Supplementary Material). Prevention activities included six prevention-truth activities (assess, examine, judge, review, scrutinize, verify) and six prevention-control activities (defend, guard, maintain, preserve, protect, resist). Promotion activities included six promotion-truth activities (discover, explore, imagine, invent, seek, wonder) and six promotion-control activities (accelerate, elevate, launch, lead, progress, propel). We also used the activity length and frequency information to ensure that the groups did not significantly differ on these dimensions. (For the results of the primary and secondary analyses conducted using this refined activity list, see Tables S6 and S7 in the Supplementary Material.)

\section{Figure 2}

Probability of Categorization as Promotion (vs. Prevention) and Log-Transformed Response Latency by Process Activity (Study 1A) 


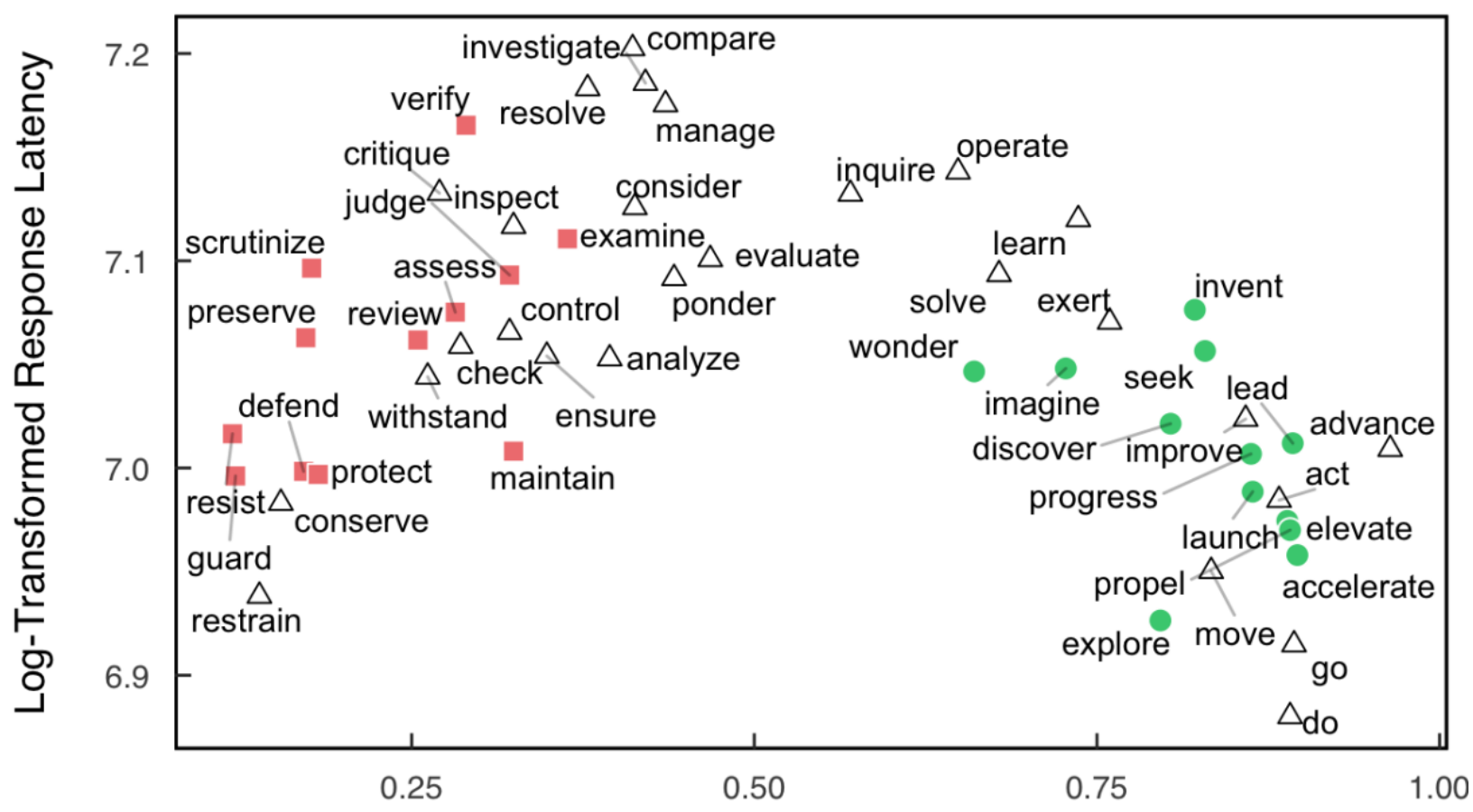

Probability of Categorization as Promotion (vs. Prevention) Status in Refined Activity List $\Delta$ Not Included $\quad$ Prevention $\bullet$ Promotion

Note. The 24 regulatory focus-specific process activities selected during refinement of the activity list are highlighted as red (dark gray) squares (prevention) and green (light gray) circles (promotion). Activities not selected during this refinement process are represented as white triangles.

\section{Discussion}

Study 1A provided initial evidence for the proposed framework of prevention- and promotion-specific goal pursuit process activities. By introducing participants to the primary distinctions between prevention and promotion and asking them to categorize process activities on this basis, we confirmed that participants' associations between these activities and regulatory focus reflected the framework. Notably, these categorization decisions were not influenced by participants' own promotion ( $v s$. prevention) pride dominance. This result is important because it 
could have been possible that participants would associate all activities with their predominant regulatory focus (e.g., if Alicia is chronically promotion-dominant, she might tend to see all goal pursuit activities as involving a promotion focus). However, this is not the pattern of results we found; instead, no general effect of promotion pride dominance emerged. This finding suggests that these associations are commonly understood and independent of individual differences in regulatory focus. This is consistent with individuals generally having available both the promotion and prevention systems (Higgins, 1997), and recognizing the difference between promotion versus prevention activities within the process of goal pursuit.

Additionally, participants categorized process activities that we hypothesized related to regulatory focus more quickly than the general truth- and control-oriented process activities we hypothesized were unrelated to regulatory focus. This response latency measure provides insight into the accessibility of these associations, suggesting that participants pause slightly when considering how to categorize activities that do not seem to clearly "fit" with a given regulatory focus. These results indicate that the associations between the constructs of prevention and promotion and the hypothesized list of prevention- and promotion-specific goal pursuit activities are more accessible than the associations between the same constructs and more general goal pursuit activities. Collectively, these findings support Hypotheses 1 and 2, providing early support for the proposed framework. As a next step, we were interested in testing the generalizability of these initial findings.

\section{Study 1B}

Study 1B aimed to replicate the findings of Study 1A and provide further evidence in support of Hypotheses 1 and 2 within a pre-registered lab study conducted on campus with a student population. By testing the framework with a sample that varied substantially from the online sample in Study 1A on a number of dimensions, including age, education, and ethnicity, 
we sought to investigate whether the associations between regulatory focus and the list of prevention versus promotion goal pursuit activities remained consistent among this different population.

\section{Method}

\section{Participants}

To determine an appropriate sample size, we used R (Version 4.0.2; R Core Team, 2019) to conduct a Bayesian equivalent of a power analysis following a simulation-based approach recommended by Kurz (2019). Given that the effects we planned to analyze in an exploratory analysis not reported here may have been smaller than the effects found in Study 1A, our goal was to obtain power of 0.90 to detect an odds ratio effect of $O R=1.50$ at $\alpha=0.05$. Our target sample size was 75 participants, and in order to account for potential exclusions, we aimed to recruit 100 participants. We also pre-registered a stopping rule indicating that if we had collected data from at least 75 participants by the end of the Fall 2019 semester, we would terminate data collection and proceed to data analysis.

Data collection took place between October 2019 and March 2020. Participants were undergraduate and post-baccalaureate students at least 18 years old at a university in the northeastern U.S. who completed the study for course credit. Although the pre-registration included a stopping rule of 75 participants, given the Covid-19 pandemic, data collection ended with a hiatus on human subjects research mandated by the university in March 2020. As a result, Study $1 \mathrm{~B}$ includes data from the 67 students recruited prior to this hiatus. Participants ranged in age from 18 to $38(M=21 ; S D=4)$. Participants were male $(36 \%)$ and female $(61 \%)$, with the remaining participants unspecified. They self-identified as White (42\%), Asian (24\%), multiracial (15\%), Hispanic (9\%), and Black (7\%). They indicated the highest level of formal education they have completed as some high school (1\%), a high school diploma (33\%), some college (51\%), an 
associate degree (4\%), and a bachelor's degree (7\%). They identified their approximate annual household income as $\$ 0-\$ 10 \mathrm{~K}(3 \%), \$ 10-\$ 20 \mathrm{~K}(3 \%), \$ 20-\$ 40 \mathrm{~K}(12 \%), \$ 40-\$ 70 \mathrm{~K}(6 \%), \$ 70-$ \$100K (13\%), \$100-\$250K (12\%), \$250-\$500K (12\%), and \$500K+ (10\%). A sensitivity analysis (G*Power; Faul et al., 2009) showed that this final sample could detect an effect size of $O R=4.78$ or larger for the effect of Hypothesized Activity Regulatory Focus (power=0.80; $\alpha=0.05$; two-tailed; conditional probability that $Y=1$ given $X=1$ under $H_{0}=0.5$; binomial $X$ distribution for which $\pi=0.5)$.

Per the pre-defined exclusion criteria, we examined the number of participants who responded that they were not native English speakers, as if this number fell below $10 \%$, we would have excluded this group. Because 11 of the 67 participants (16.4\%) reported a native language other than English, these participants were not excluded from the primary analyses. As described in the study's pre-registration, we also conducted all analyses excluding this group and found very similar results (see Tables S14 and S15 in the Supplementary Material).

As in Study 1A, we excluded activity categorization decisions for which the response latency was less than $250 \mathrm{~ms}$ or greater than $10,000 \mathrm{~ms}$. After removing these observations, the dataset included 2,703 total activity categorization decisions, with a mean of 40.03 categorization decisions per participant $(S D=1.60)$.

\section{Materials and Measures}

Activity List. In Study 1B, the activity list comprised 39 activities in total. Among these activities, 36 were drawn from the activity list used in Study 1A, including the 24 promotion- and prevention-specific goal pursuit process activities adopted from the refined activity list and six general truth- and control-oriented process activities (along with six practice verbs reflecting the acquisition of promotion- and prevention-specific outcomes). Additionally, three more general activities (sleep, shower, communicate) were included. For a complete list of these activities, see 
Table S8 in the Supplementary Material. As in Study 1A, the regulatory focus of each of these activities was dummy-coded ( $0=$ prevention; $1=$ promotion $)$. Based on these hypotheses, we manipulated activity regulatory focus (i.e., our primary independent variable) on a withinparticipants basis in the activity sorting task described next.

Activity Sorting Task. Participants categorized each of the verbs in the activity list as prevention or promotion activities within a computerized sorting task very similar to the task administered in Study 1A, with some minor changes. Given some feedback from participants that the sorting task in Study 1A could have been administered more quickly, in Study 1B, the intertrial interval was set to $1,000 \mathrm{~ms}$. Additionally, based on this same feedback, the sorting task in Study 1B only comprised one group of trial blocks, rather than two. Like in Study 1A, when participants began the sorting task, the placement of the "prevention" and "promotion" category labels was counterbalanced; however, given that this study only contained one group of trial blocks, this randomly assigned category placement did not change midway through the task. Finally, the number of trials was significantly reduced, as a result of the smaller activity list and the inclusion of only one group of trial blocks. After the first practice block consisting of six trials, which was intended to familiarize participants with the logistics of the task, the second block involved categorizing the 33 process activities of interest as prevention or promotion.

Additionally, for exploratory purposes, participants performed a second activity sorting task in which they were asked to categorize activities as reflecting truth versus control motivation, rather than prevention versus promotion. (The results of this exploratory task are not discussed further here.)

Regulatory Focus Pride. As in Study 1A, to measure chronic historical effectiveness (i.e., "pride") in the domains of prevention and promotion, we administered the RFQ (Higgins et al., 2001). After determining mean endorsement levels for each participant $\left(M_{\text {prevention }}=3.36\right.$; 
$\left.S D_{\text {prevention }}=0.92 ; \alpha_{\text {prevention }}=0.84 ; M_{\text {promotion }}=3.71 ; S D_{\text {promotion }}=0.61 ; \alpha_{\text {promotion }}=0.64\right)$, we calculated promotion ( $v s$. prevention) pride dominance scores by subtracting each participant's prevention pride score from their promotion pride score $(M=0.36 ; S D=1.14)$. These promotion pride dominance scores were mean-centered for use in the regression analyses.

Further Measures. The only new measure administered in Study 1B was the Regulatory Focus Strength measure (Higgins et al., 1997), which was included for exploratory purposes. Whereas the RFQ assesses participants chronic historical effectiveness (i.e., pride) in the domains of prevention and promotion, the Regulatory Focus Strength measure is an idiographic measure that captures the accessibility of participants' ought (i.e., prevention) versus ideal (i.e., promotion) goals. Participants listed four attributes describing each of these types of goals in a pseudo-random order, while the computer captured their response latency in submitting these goals. Additionally, participants rated the degree to which they ideally would possess or ought to possess the attribute, as well as the degree to which they actually possess the attribute, on a fourpoint scale (1=slightly to $4=$ extremely). Response latencies were also captured when making these ratings. As per Higgins, Shah, and Friedman (1997), these response latencies were logtransformed to normalize the distributions. Then, prevention and promotion strength scores were calculated by summing the nine log-transformed response latencies associated with the first three oughts $(\alpha=0.77)$ and first three ideals $(\alpha=0.79)$, respectively. Finally, these scores were multiplied by -1 so that higher scores indicated greater strength $\left(M_{\text {prevention }}=-4.44\right.$; $\left.S D_{\text {prevention }}=1.13 ; M_{\text {promotion }}=-4.43 ; S D_{\text {promotion }}=1.13\right)$.

Like in Study 1A, to control for any potential effects of stimulus length or frequency, we measured these variables for all activities. We also measured participants' assessment $(M=4.46$; $S D=0.66 ; \alpha=0.76)$ and locomotion $(M=4.37 ; S D=0.69 ; \alpha=0.83)$ orientations using the RMQ (Kruglanski et al., 2000). Additionally, we administered the same exploratory measures probing 
participants' experiences in completing the prevention versus promotion activity sorting task as in Study 1A. Finally, participants completed a second set of questions that asked about their experience in completing the exploratory truth versus control activity sorting task. Although responses to these further measures are not discussed here, the data are available on OSF.

\section{Procedure}

After consenting to participate, participants completed three motivational measures: the Regulatory Focus Strength measure, the RMQ, and the RFQ. Then, after learning about the distinction between prevention and promotion motivation using the same training administered in Study 1A, participants completed the prevention versus promotion activity sorting task. Next, they completed a similar exploratory activity sorting task in which they categorized the verbs based on their associations with truth versus control. After finishing these sorting tasks, participants completed the exploratory items intended to probe their categorization experience. Finally, after optionally providing brief demographic information, participants were debriefed, thanked, and compensated.

\section{Results}

\section{Analytic Strategy}

As in Study 1A, our primary analysis examined whether our predictions about the hypothesized regulatory focus of each prevention or promotion process activity were reflected in participants' categorization decisions. To conduct this analysis, we selected the subset of the data comprising only the 24 activities we hypothesized reflected prevention- or promotion-specific processes $(N=1,604)$ and analyzed these categorization decisions within a Bayesian multilevel binomial logistic regression model specified identically to the model in Study 1A. To conduct the secondary analysis testing whether verbs that we hypothesized were prevention- or promotionspecific process activities were categorized more quickly than general process activities that we 
hypothesized were not associated with a particular regulatory focus, we analyzed categorization response latencies for all 33 process activities $(N=2,204)$ within a Bayesian multilevel multiple regression model identical to the model specified in Study 1A. (For descriptive statistics and correlations among all variables included within these models, see Table S9 in the Supplementary Material.)

\section{Activity Categorization Decisions}

Among the categorization decisions for all goal pursuit process activities (i.e., regulatory focus-specific and general process activities), on average, $45.24 \%$ of activities were categorized as prevention and $54.76 \%$ as promotion. Among the decisions made with respect to the proposed list of prevention- and promotion-specific goal pursuit process activities, a similar pattern emerged: an average of $43.89 \%$ of activities were categorized as prevention and $56.11 \%$ as promotion. By estimating the intercept within a Bayesian multilevel logistic regression model including a fixed intercept as well as random intercepts of participant and activity, we determined that the probability of categorizing our hypothesized regulatory focus-specific process activities as promotion ( $\log$ odds of 0.99 units; $95 \% \mathrm{CI}:-0.72,2.70$; probability of $72.91 \%$; $95 \% \mathrm{CI}$ : $32.65 \%, 93.70 \%$ ) did not differ significantly from chance (i.e., a probability of 50\%).

\section{Activity Categorization Response Latency}

Among all categorization decisions (i.e., for all process activities), on average, activities were categorized in 1.38 seconds, and hypothesized prevention- and promotion-specific goal pursuit process activities were categorized in 1.26 seconds.

\section{Primary Analysis: Do Our Hypotheses About Whether Each Process Activity Reflects a} Promotion (vs. Prevention) Focus Predict Participants' Categorization Decisions?

To test if our hypotheses about the process activities' regulatory focus predicted participants' categorization decisions, we examined the model output from the Bayesian 
multilevel logistic regression analysis (see the upper panel of Table 4 and Figure 3). The estimated effect of hypothesized activity regulatory focus was $7.76 \log$ odds units $(O R=$ $2,344.90)$, with a $95 \%$ credible interval that did not include zero $(6.33,9.46)$. In line with Hypothesis 1, this credible interval suggests that there is an association between the promotion versus prevention focus of goal pursuit process activities and participants' categorization decisions. Transformed into probabilities, the model predicts that activities that we hypothesized were associated with prevention had a $93.40 \%$ probability of being categorized as prevention, and activities that we hypothesized were associated with promotion had a $99.40 \%$ probability of being categorized as promotion.

\section{Table 4}

Parameter Estimates for Multilevel Binomial Logistic Regression Analysis Predicting Process Activity Categorization and Multilevel Multiple Regression Analysis Predicting Log-Transformed Response Latency (Study 1B; Fixed Effects Only)

\begin{tabular}{lrrrr}
\hline & & & \multicolumn{2}{c}{$95 \%$ CI } \\
\cline { 3 - 5 } Effect & Estimate & Post.SD & Lower & Upper \\
\hline DV: Activity Categorization (0=Prev.; 1=Prom.) & & & & \\
Intercept & -2.65 & 0.48 & -3.68 & -1.77 \\
Hypothesized Activity RF (0=Prev.; 1=Prom.) & $\mathbf{7 . 7 6}$ & $\mathbf{0 . 7 9}$ & $\mathbf{6 . 3 3}$ & $\mathbf{9 . 4 6}$ \\
Promotion (vs. Prevention) Pride Dominance & 0.02 & 0.17 & -0.31 & 0.36 \\
Trial & 0.14 & 0.14 & -0.15 & 0.42 \\
Order of Category Labels & 0.51 & 0.35 & -0.17 & 1.20 \\
Frequency of Use in English Language & 0.24 & 0.48 & -0.71 & 1.19 \\
Length & 0.48 & 0.45 & -0.40 & 1.40 \\
DV: Log-Transformed Response Latency & & & & \\
Intercept & 7.33 & 0.06 & 7.21 & 7.45 \\
Hypothesized RF-Specific Activity (0=No; 1=Yes) & $-\mathbf{0 . 2 8}$ & $\mathbf{0 . 0 6}$ & $-\mathbf{0 . 4 0}$ & $\mathbf{- 0 . 1 5}$ \\
Promotion (vs. Prevention) Pride Dominance & -0.02 & 0.03 & -0.07 & 0.03 \\
Trial & -0.07 & 0.01 & -0.10 & -0.05 \\
Order of Category Labels & -0.08 & 0.06 & -0.19 & 0.03 \\
Frequency of Use in English Language & -0.03 & 0.03 & -0.08 & 0.02 \\
Length & -0.03 & 0.03 & -0.08 & 0.03 \\
\hline
\end{tabular}


Note. $\mathrm{RF}=$ Regulatory Focus; Prom.=Promotion; Prev.=Prevention. Effects for the predictor of interest are shown in bold. For the complete results of these analyses, including random effects, see Tables S10 and S11 in the Supplementary Material.

\section{Figure 3}

Probability of Categorization as Promotion (vs. Prevention) as a Function of Hypothesized Process Activity Regulatory Focus (Study 1B)

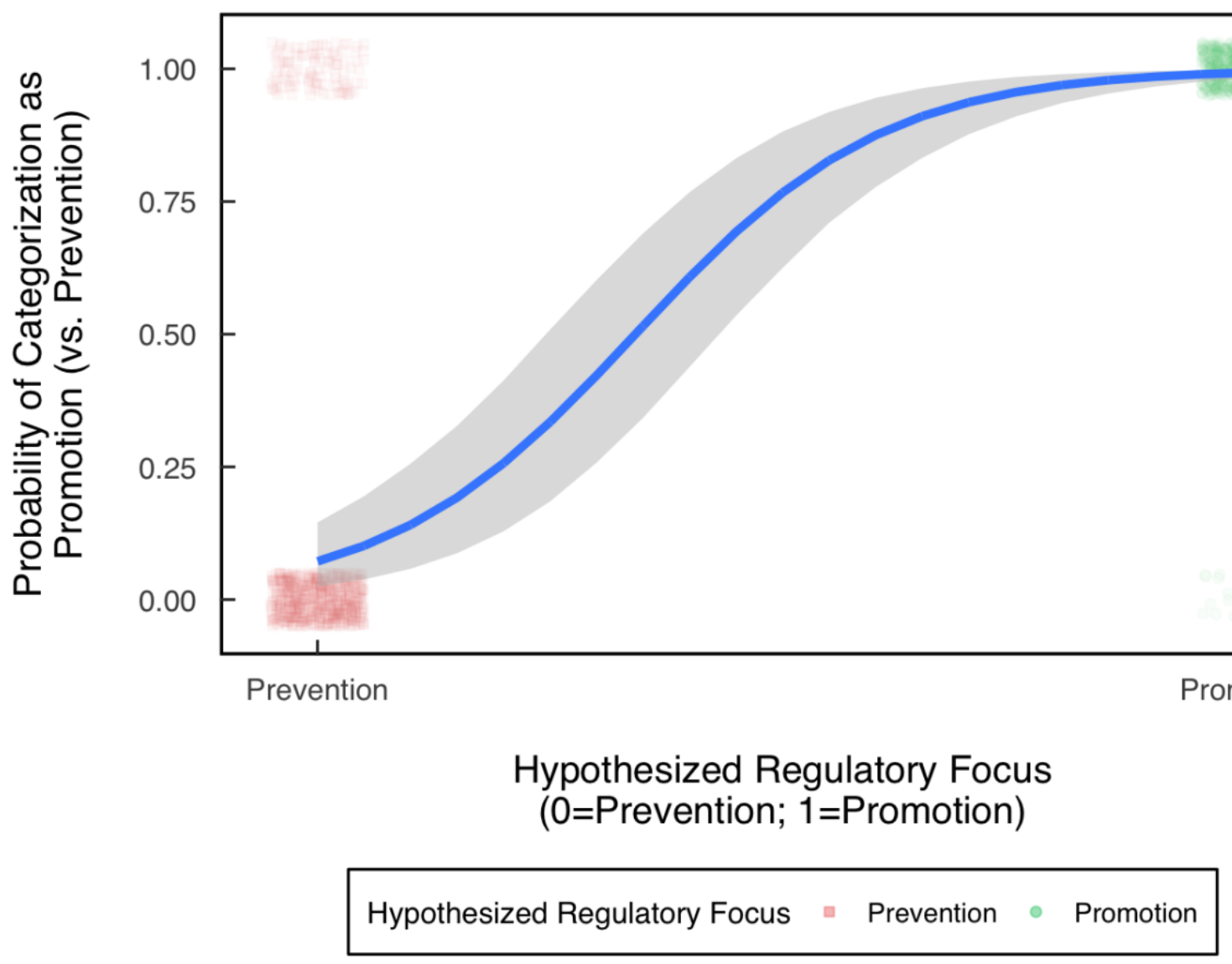

Note. Model-predicted probability of categorization as promotion ( $v s$. prevention) as a function of hypothesized process activity regulatory focus, at typical levels of promotion pride dominance, trial, order of label presentation, activity frequency of use in the English language, and activity length. The red (dark gray) squares represent categorization decisions for activities hypothesized to reflect prevention processes. The green (light gray) circles represent categorization decisions 
for activities hypothesized to reflect promotion processes. Shaded region represents $95 \%$ credible interval.

Additionally, like in Study 1A, we were interested in examining whether participants' categorization of any given process activity as promotion or prevention was associated with their promotion ( $v s$. prevention) pride dominance. As indicated in the upper panel of Table 4, the estimated effect of promotion pride dominance on activity categorization is 0.02 , with a $95 \%$ credible interval that includes zero $(-0.31,0.36)$. This credible interval indicates that we cannot infer any association between individual differences in promotion pride dominance and participants' categorization decisions. Furthermore, no other fixed effects in this primary analysis had a credible interval excluding zero.

\section{Secondary Analysis: Are Activities Hypothesized to Be Associated with a Specific Regulatory} Focus Process Categorized More Quickly Than Process Activities Without These Hypothesized Associations?

To test if our hypotheses about the process activities' associations with regulatory focus (or lack thereof) predicted how quickly participants made their categorization decisions, we examined the model output from the Bayesian multilevel multiple regression analysis (see the lower panel of Table 4). The estimated effect of this hypothesized association with regulatory focus is $-0.28 \log$-transformed milliseconds, with a $95 \%$ credible interval that did not include zero $(-0.40,-0.15)$. In line with Hypothesis 2 , these results indicate that participants were quicker to categorize activities that we hypothesized were ( $v s$. were not) associated with a specific regulatory focus process. The model predicts that activities that we hypothesized were associated with a specific regulatory focus process would be categorized in 1.15 seconds, and activities that we hypothesized were not associated with a specific regulatory focus process would be 
categorized in 1.53 seconds. To visualize these differences in response latency as well as activity categorization, see Figure 4.

\section{Figure 4}

Probability of Categorization as Promotion (vs. Prevention) and Log-Transformed Response Latency by Activity (Study 1B)

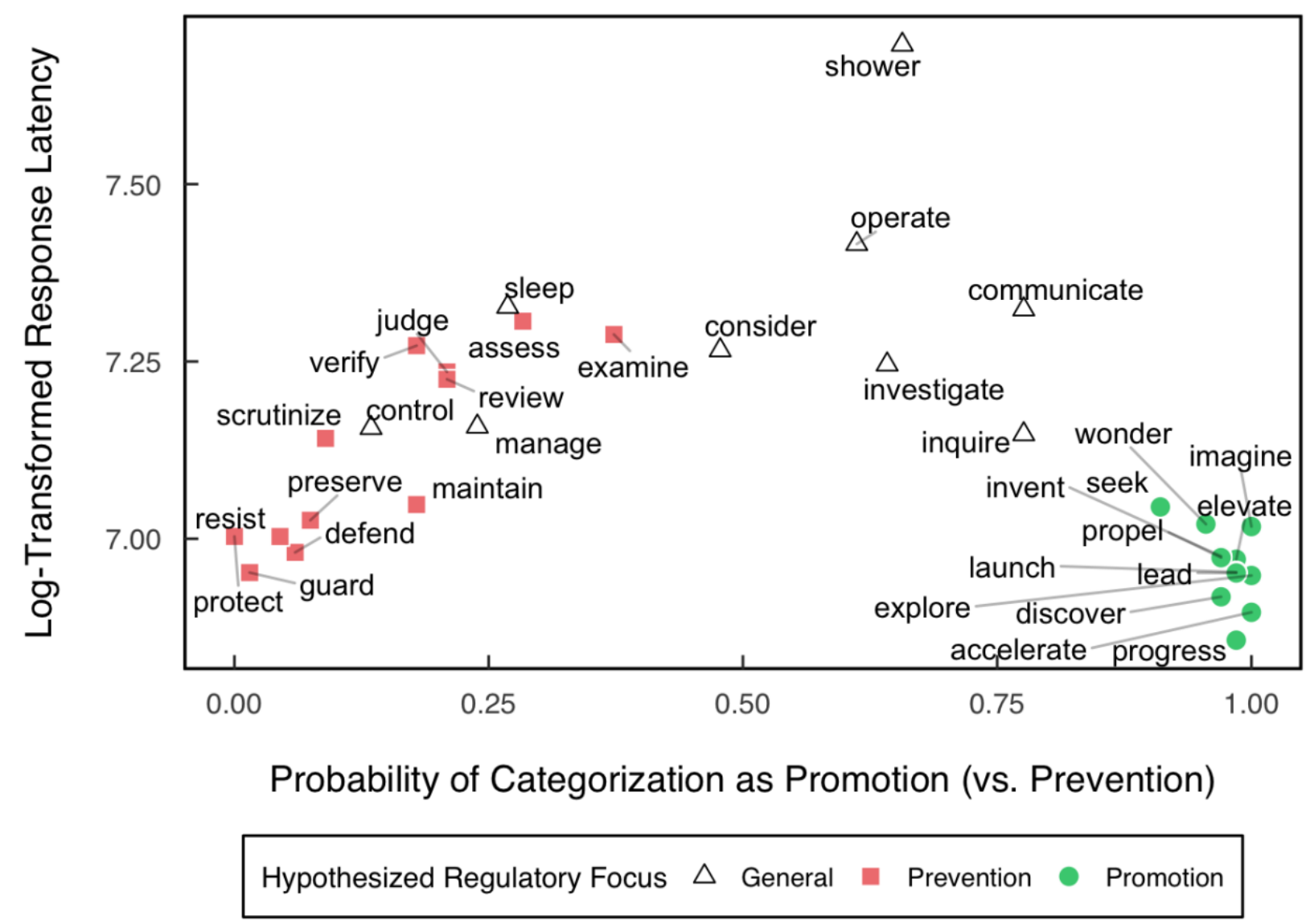

Note. The 24 process activities hypothesized to relate to regulatory focus are highlighted as red (dark gray) squares (prevention) and green (light gray) circles (promotion). Activities hypothesized not to relate to a specific regulatory focus are represented as white triangles.

Additionally, this analysis allowed us to test if participants' response latencies in categorizing these process activities were associated with their promotion ( $v s$. prevention) pride dominance. As indicated in the lower panel of Table 4, the estimated effect of promotion pride 
dominance is -0.02 , with a $95 \%$ credible interval that includes zero $(-0.07,0.03)$. These results suggest no association between individual differences in promotion pride dominance and participants' categorization speed. Like in Study 1A, trial was also associated with participants' response latency; however, whereas activities' frequency of use in the English language was associated with faster predictions in Study 1A, we did not detect such an effect in Study 1B. ${ }^{1}$

\section{Discussion}

Study 1B provided additional evidence in support of Hypotheses 1 and 2 as well as the proposed framework of prevention- and promotion-specific goal pursuit process activities. The results were highly consistent with the results of Study 1A, indicating that (a) participants' categorization decisions reflected the framework, (b) participants categorized activities that we hypothesized were related to regulatory focus processes more quickly than the general truth- and control-oriented process activities that we hypothesized were unrelated to regulatory focus, and (c) individual differences in promotion pride dominance had no general effect on categorization decisions or speed.

Collectively, the results of Studies 1A and 1B indicate that the associations between regulatory focus and the hypothesized list of prevention- and promotion-specific goal pursuit process activities are highly accessible and commonly available among samples including MTurk workers and university students. Given the consistency in results across both samples, these findings suggest that these associations may be fundamental in nature. As a next step, we followed Eitam and Higgins' (2010) proposal that the accessibility of a representation is

\footnotetext{
${ }^{1}$ For exploratory purposes, we also conducted these primary and secondary analyses with promotion (vs. prevention) strength dominance, rather than promotion ( $v s$. prevention) pride dominance, included as a covariate. The results of these exploratory analyses were consistent with the results of the analyses reported above; both key effects of interest remained similar in size, and promotion strength dominance was not associated with participants' categorization decisions or response latency. See Tables S12 and S13 in the Supplementary Material for the complete results of these exploratory analyses.
} 
influenced by its motivational relevance (Hypothesis 3). For example, if the activity scrutinize is fundamentally associated with the prevention system, will it be more accessible to a person strongly ( $v s$. weakly) motivated by prevention? As described briefly earlier, in Studies 2A and 2B, we operationalized accessibility as activities' output primacy (Higgins, 1996) within an unaided recall task. Study 2 A examined how output primacy related to the interaction between measured regulatory focus strength and motivationally relevant ( $v s$. irrelevant) process activities, and Study 2B examined how manipulated regulatory focus strength influenced the output primacy of motivationally relevant ( $v s$. irrelevant) process activities.

\section{Study 2A}

Study 2A served as our first investigation of Hypothesis 3. Specifically, the study aimed to test whether the associations between the prevention and promotion systems and various activities in the goal pursuit process extend to the accessibility of these activities for a given individual. After completing measures examining their chronic motivational orientations, participants were presented with 36 different activities and asked to rate their importance. Then, following a brief distraction, participants were surprised with an unaided recall task in which they were asked to list as many of the activities rated earlier as possible. We then examined the order in which they recalled these activities ("output rank") as the primary dependent measure. We hypothesized that participants' regulatory focus strength would interact with each activity's regulatory focus to predict output rank, such that participants with stronger promotion ( $v s$. prevention) strength would tend to output goal pursuit activities associated with promotion ( $v s$. prevention) processes earlier.

\section{Method}

\section{Participants}


Similar to Study 1A, although our analytic approach is primarily Bayesian, we conducted a traditional power analysis to determine an appropriate sample size for this first study as, given the novelty of this research, we lacked the prior knowledge needed to appropriately proceed with a simulation-based approach. We aimed to detect a medium effect of $f^{2}=0.15$ (Cohen, 1988) with power of 0.80 . This power analysis ( $\mathrm{G}^{*}$ Power; Faul et al., 2009; $\alpha=0.05$; two-tailed; testing one of six total predictors) indicated that 55 participants would be required. In order to account for potential exclusions, we set the target sample size at 75 participants.

Data collection took place in July 2019. We recruited 75 native English speaking U.S. MTurk workers at least 18 years old. All participants were paid \$1.75. As in Study 1A, we examined the dataset for fraudulent responses based on participant IP addresses using R (Version 4.0.2; R Core Team, 2019) and the R package rIP (Version 1.2.0; Kennedy \& Waggoner, 2019). This review revealed that four responses were submitted by IP addresses flagged as fraudulent, so these responses were excluded. Additionally, we examined the number of participants who disregarded the recruitment criteria and participated despite not being native English speakers. Only one of the remaining participants reported a native language other than English, and because the percentage of non-native English speaking participants (1.4\%) fell below our 10\% threshold, this participant was excluded from the primary analysis.

Among the remaining 70 participants, four participants failed to properly complete the measure that collected the primary predictor variables (Regulatory Focus Strength), and nine participants failed to recall any activities from the framework at the end of the study (i.e., the primary dependent variable). These exclusions left a sample of 57 participants. These participants ranged in age from 21 to $64(M=36 ; S D=11)$. Participants were male $(53 \%)$ and female (47\%). They self-identified as White (72\%), Asian (9\%), Hispanic (7\%), Black (7\%), and multiracial (5\%). They indicated the highest level of formal education they have completed as 
some high school (2\%), a high school diploma (21\%), some college (19\%), an associate degree (18\%), a bachelor's degree (35\%), and a master's degree (5\%). They identified their approximate annual household income as \$10-\$20K (9\%), \$20-\$40K (23\%), \$40-\$70K (39\%), \$70-\$100K (18\%), and $\$ 100-\$ 250 \mathrm{~K}(11 \%)$. A sensitivity analysis ( $\mathrm{G}^{*}$ Power; Faul et al., 2009) showed that this final sample could detect an effect size of $f^{2}=0.14$ or larger for the critical interaction between Activity Regulatory Focus and participants' Regulatory Focus Strength Dominance (power=0.80; $\alpha=0.05$; two-tailed; testing one of six total predictors).

The dataset included 398 total outputted activities. Of these observations, 254 outputted activities were regulatory focus-specific processes from the hypothesized framework. Among the sample of participants who outputted at least one of the 24 prevention and promotion goal pursuit process activities, the number of activities outputted ranged from 1 to $10(M=4.46, S D=2.46)$. For exploratory purposes, we also conducted the primary analysis on a subset of the data including only the 41 participants who outputted at least one prevention process activity and one promotion process activity (total number of regulatory focus-specific process activities outputted among this group=221). The results of this exploratory analysis can be found in Table S21 in the Supplementary Material.

\section{Materials and Measures}

Regulatory Focus Strength. Promotion and prevention strength were assessed using the same idiographic, response latency-based Regulatory Focus Strength measure (Higgins et al., $1997)$ administered in Study $1 \mathrm{~B}\left(M_{\text {prevention }}=-3.09 ; S D_{\text {prevention }}=1.54 ; \alpha_{\text {prevention }}=0.81 ; M_{\text {promotion }}=-\right.$ $\left.3.42 ; S D_{\text {promotion }}=1.47 ; \alpha_{\text {promotion }}=0.83\right)$. We then calculated promotion ( $v s$. prevention) strength dominance scores, as like promotion and prevention pride, these orientations involve different motivational preferences and, as a result, competing accessibility effects. We calculated these dominance scores by subtracting each participant's prevention strength score from his/her 
promotion strength score $(M=-0.33 ; S D=0.89)$. Finally, these promotion strength dominance scores were mean-centered for use in the regression analyses so the reference condition would reflect the typical level of promotion strength dominance. To do so, we subtracted each participant's promotion strength dominance score from the promotion strength dominance grand mean.

Additionally, for exploratory purposes, participants' promotion and prevention strength scores were mean-centered so the reference conditions for these variables would reflect typical levels of promotion and prevention strength. To do so, we subtracted each participant's promotion strength score from the promotion strength grand mean, and prevention strength score from the prevention strength grand mean. We also scaled these mean-centered scores such that $S D=1$. The results of exploratory analyses using these independent scores were very similar to the results of analyses using the dominance score (see Table S22 in the Supplementary Material).

Activity List. In Study 2A, the activity list comprised 36 activities in total. Among these activities, 30 process activities reflected the activity list refined in Study 1A and used in Study $1 \mathrm{~B}$, including the same 24 promotion- and prevention-specific goal pursuit process activities and six general truth- and control-oriented process activities. (No verbs reflecting the acquisition of promotion- and prevention-specific outcomes were included, as these stimuli were only included in Studies 1A and 1B for use in the practice round of the sorting task, and Study 2A involved no such task.) Further, in addition to the three general process activities included in the activity list used in Study 1B, three more general activities (exercise, read, pray) were included to help ensure that the study's activity importance rating task felt like a comprehensive review of all kinds of everyday activities. For a complete activity list, see Table S16 in the Supplementary Material. Most relevant to our investigation were the 12 activities hypothesized to relate to prevention processes and the 12 activities hypothesized to relate to promotion processes. The 
regulatory focus of each of these process activities was effect-coded $(-0.5=$ prevention; $+0.5=$ promotion $)$ and manipulated on a within-participants basis in the activity importance rating task.

Activity Importance Rating Task. In order to present all 36 activities to participants a first time, participants were asked to rate the importance of each of these activities. Instructions described that the research team was interested in how important participants considered it to be that they engaged in each activity in their own lives. Each activity was presented and rated on a separate page of the questionnaire, which facilitated the collection of activity-specific response latency data for exploratory purposes. Participants responded to the question "How important is it that you engage in this activity?" on a 101 -point sliding scale $(0=$ not at all important to 5=extremely important). Activities were presented in a random order.

Distractor Task. Because we were interested in testing the accessibility of these activities at the end of the study, we briefly distracted participants with the intention of clearing the activities from working memory. To serve as this distraction, participants completed ten visual pattern-completion items from Raven's Progressive Matrices (Raven \& Raven, 2003), a test of general cognitive ability. On average, this distraction lasted 2.49 minutes.

Unaided Recall Task. To measure the accessibility (i.e., output rank) of each activity, instructions reminded participants that earlier they rated the importance of 36 different activities, and asked them to recall as many of the activities as they could and to list them in a series of 36 text boxes on the page. (The instructions also described that if participants did not remember all 36 activities, it is OK to leave some of the text boxes blank.) Data from this task were handcoded by research assistants to capture activities recalled with minor misspellings (e.g., "perserve" instead of "preserve") as well as activities recalled in the form of a participle (e.g., "preserving"). For each recalled activity, coders noted the output rank of the activity - that is, 
whether the activity was listed in the first text box (i.e., an output rank of 1), the second text box (i.e., an output rank of 2), and so on. These output ranks were then log-transformed to normalize the distributions and then multiplied by -1 so that higher scores indicated greater output primacy (i.e., accessibility). Then, these output rank data were dummy-coded to produce a binary measure of output $(0=$ not recalled; $1=$ recalled $)$. An output total score was calculated by summing the number of activities each participant recalled and rescaling these sums $(M=0 ; S D=1)$.

Further Measures. As in Studies 1A and 1B, to control for any potential effects of stimulus length or frequency, we measured each of these variables for all activities. We also measured participants' prevention pride $(M=3.45 ; S D=0.84 ; \alpha=0.87)$ and promotion pride $(M=3.42 ; S D=0.68 ; \alpha=0.77)$ scores using the RFQ (Higgins et al., 2001), and their assessment $(M=3.77 ; S D=0.91 ; \alpha=0.86)$ and locomotion $(M=4.34 ; S D=0.72 ; \alpha=0.87)$ orientations using the RMQ (Kruglanski et al., 2000).

\section{Procedure}

After consenting to participate, participants completed three motivational measures: the Regulatory Focus Strength measure, followed by the RMQ and the RFQ. Next, participants proceeded to the activity importance rating task, thus encountering all 36 activities for the first time. Then, participants completed the brief distractor, which was followed by the unaided recall task probing the output primacy of the various activities. After completing these tasks, participants optionally provided brief demographic information and were debriefed, thanked, and compensated.

\section{Results}

\section{Analytic Strategy}

Because we hypothesized that the accessibility of prevention versus promotion process activities would be associated with participants' own regulatory focus strength, the primary 
analysis examined whether the interaction between the hypothesized regulatory focus of a given process activity and each participant's promotion ( $v s$. prevention) strength dominance predicted the log-transformed output rank of the activity. In line with our analytic approach in Studies $1 \mathrm{~A}$ and $1 \mathrm{~B}$, we selected the subset of data comprising only activities we hypothesized to reflect prevention- or promotion-specific processes $(N=254)$ and analyzed these data within a Bayesian multilevel regression model. Participants' log-transformed output rank (i.e., our primary dependent variable) was regressed on the hypothesized activity regulatory focus (effect-coded:$0.5=$ prevention $;+0.5=$ promotion $)$, the participant's mean-centered promotion ( $v s$. prevention) strength dominance, and the interaction of interest between activity regulatory focus and promotion strength dominance. Other covariates included as fixed effects were activity frequency and activity length (both rescaled: $M=0 ; S D=1$ ). Random effects included in the model were random intercepts of participant and activity; random slopes of activity frequency and length by participant; and random slopes of promotion strength dominance by activity.

Importantly, we detected two sets of substantial correlations between participants' output total and both (a) participants' promotion ( $v s$. prevention) strength dominance $(r=0.24$; $95 \%$ CI: $0.01,0.46)$, a key predictor variable, and (b) log-transformed output rank $(r=-0.78 ; 95 \% \mathrm{CI}$ :$0.87,-0.67)$, the dependent variable. Given these correlations, we were interested in more precisely isolating the effects of the interaction between regulatory focus strength and the hypothesized regulatory focus on output rank, beyond any differences in their output total. As a result, although not initially planned, we re-conducted the primary analysis with output total included as a covariate. Using R (Version 4.0.2; R Core Team, 2019) and the R package brms (Version 2.13.5; Bürkner, 2017, 2018) and following a validated model comparison procedure (Vehtari et al., 2017), we conducted a "leave-one-out" cross-validation analysis comparing the same model with and without the inclusion of output total. Because this model comparison 
procedure indicated that the model with this covariate has greater expected predictive accuracy, the results of this revised model are included below. (The model comparison analysis and all results using the original model without output total included as a covariate are provided in and following Table S19 in the Supplementary Material.) For descriptive statistics and correlations among all variables included within these models, see Table S17 in the Supplementary Material.

Additionally, because we hypothesized that the most accessible activities would be recalled earliest in the memory task, we also examined a subset of the data that was recalled earliest in the study. As detailed further below, these analyses indicated that the focal interaction is most strongly associated with accessibility (i.e., output rank) when including only the first 13 activities that participants recalled within the unaided recall task. As a result, we report the results of the original analyses including all recalled regulatory focus-specific process activities as well as the results of analyses including only the regulatory focus-specific process activities included in participants' first 13 recalled activities below.

Finally, although the primary analysis contains data for all participants, given that these analyses differentiate between the average output rank for prevention versus promotion process activities, we also conducted these analyses only including data for participants who remembered at least one prevention activity and at least one promotion activity. The results of these analyses are presented in Table S21 in the Supplementary Material.

\section{Output Total: Number of Activities Recalled}

On average, participants recalled 6.98 total activities $(S D=3.28)$ in the unaided recall task. Among these activities, participants recalled an average of 4.46 activities that we hypothesized were associated with prevention or promotion processes $(S D=2.46)$, comprising an average of 2.51 prevention activities $(S D=1.64)$ and 1.95 promotion activities $(S D=1.41)$. By estimating the difference between the number of prevention versus promotion process activities recalled within 
a Bayesian linear regression model, we determined that participants recalled 0.56 (95\% CI: 0.08, 1.06) more prevention process activities than promotion process activities.

\section{Output Rank: Primacy of Activities Recalled}

Across participants, the average log-transformed output rank among the recalled activities in the unaided recall task was $-1.37(S D=0.74)$. Prevention process activities $(M=-1.35$; $S D=0.68)$ tended to be recalled slightly earlier than promotion process activities $(M=-1.40$; $S D=0.72$ ). However, by estimating the difference between the log-transformed output rank of promotion versus prevention process activities within a Bayesian linear regression model, we determined that this difference did not differ from zero (95\% CI: $-0.30,0.15)$.

Primary Analysis: Does the Interaction Between Participants' Regulatory Focus Strength and Activity Regulatory Focus Predict the Output Rank of Activities in the Framework?

We hypothesized that the interaction between participants' promotion (vs. prevention) strength dominance and the regulatory focus of each prevention- or promotion-specific process activity in the hypothesized framework would predict that activity's log-transformed output rank. To investigate this question, we examined the model output from the Bayesian multilevel regression analysis (see Table 5). The effect size for this interaction was 0.27 units, with a $95 \%$ credible interval that did not include zero $(0.05,0.49)$. Based on this credible interval and the positive direction of the effect, and in line with Hypothesis 3, these results suggest that the interaction between these variables predicts regulatory focus-specific process activities' output rank. With each additional point of promotion ( $v s$. prevention) strength dominance, participants tended to output promotion ( $v s$. prevention) process activities earlier, indicating that this increase in motivational predominance predicts an increase in the accessibility of motivationally relevant activities. (To visualize the model's predicted output rank for promotion versus prevention process activities as a function of promotion strength dominance, see Figure 5.) Finally, output 
total was the only other predictor of log-transformed output rank $(b=-0.29 ; 95 \% \mathrm{CI}:-0.37,-$ $0.21)$.

Table 5

Parameter Estimates for Multilevel Multiple Regression Analysis Predicting Log-Transformed Output Rank of Regulatory Focus-Specific Process Activities (Study 2A; Fixed Effects Only)

\begin{tabular}{lrrrr}
\hline & & & \multicolumn{2}{c}{$95 \%$ CI } \\
\cline { 4 - 5 } Effect & Estimate & Post.SD & Lower & Upper \\
\hline DV: Log-Transformed Output Rank (Higher=More & Accessible $)$ & & & \\
Intercept & -1.40 & 0.08 & -1.55 & -1.25 \\
Hypothesized Activity RF (-0.5=Prev.; +0.5=Prom.) & -0.06 & 0.14 & -0.34 & 0.23 \\
Promotion (vs. Prevention) Strength Dominance & 0.00 & 0.06 & -0.12 & 0.12 \\
Output Total & -0.29 & 0.14 & -0.37 & -0.21 \\
Frequency of Use in English Language & 0.08 & 0.13 & -0.17 & 0.33 \\
Length & 0.05 & 0.10 & -0.14 & 0.24 \\
Activity RF×Prom. (vs. Prev.) Strength Dominance & $\mathbf{0 . 2 7}$ & $\mathbf{0 . 1 1}$ & $\mathbf{0 . 0 5}$ & $\mathbf{0 . 4 9}$ \\
\hline
\end{tabular}

Note. $\mathrm{RF}=$ Regulatory Focus; Prom.=Promotion; Prev.=Prevention. Effects for the predictor of interest are shown in bold. For the complete results of this analysis, including random effects, see Table S18 in the Supplementary Material.

\section{Figure 5}

Log-Transformed Output Rank for Promotion Versus Prevention Process Activities as a Function of Promotion Strength Dominance (Study 2A) 


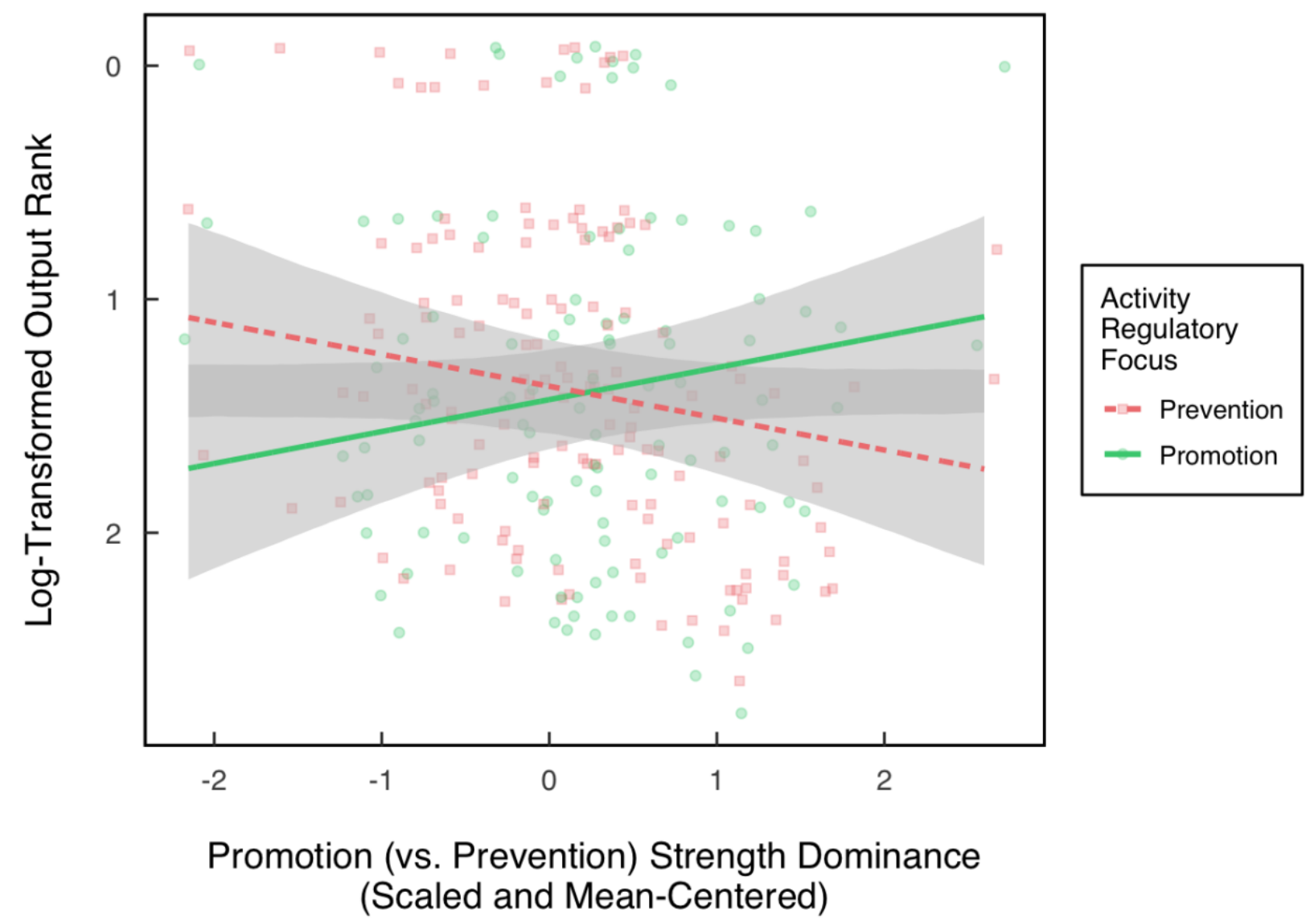

Note. Model-predicted log-transformed output rank for promotion versus prevention process activities as a function of promotion strength dominance, at typical levels of output total, activity frequency of use in the English language, and activity length. The log-transformed output rank values on the $\mathrm{Y}$-axis have been reversed so that output ranks higher in the plot indicate greater accessibility. The red (dark gray) squares and dashed line represent outputted prevention process activities. The green (light gray) circles and solid line represent outputted promotion process activities. Shaded regions represent $95 \%$ credible intervals.

Exploratory Analysis: When Examining Activities with the Greatest Output Primacy, Does the Interaction Between Participants' Regulatory Focus Strength and Activity Regulatory Focus Predict the Output Rank of Activities in the Framework? 
Accessibility measures often use output primacy to determine which participant responses are included in a given average score. One such example is the Regulatory Focus Strength measure (Higgins et al., 1997), which examines whether prevention-related oughts or promotionrelated ideals are submitted and rated more quickly. When calculating prevention and promotion strength scores, researchers examine only the response latencies for only the first nine attributes and ratings that participants submit earliest in the task, and they discard the response latencies for attributes and ratings submitted later. With this approach in mind, we conducted a number of exploratory analyses examining whether the interaction effect of interest would be more visible when testing only an early subset of the data. We found that examining the first 13 recalled activities provided a slightly clearer view of this effect, with an effect size for the key interaction of 0.28 units and a $95 \%$ credible interval that did not include zero $(0.06,0.50$; see Table 6$)$. These results suggest that the interaction between these variables predicts regulatory focus-specific process activities' output rank.

\section{Table 6}

Parameter Estimates for Multilevel Multiple Regression Analysis Predicting Log-Transformed Output Rank of Regulatory Focus-Specific Process Activities (Study 2A; Data from First 13 Outputted Activities Only; Fixed Effects Only)

\begin{tabular}{lrrrr}
\hline & & & \multicolumn{2}{c}{$95 \%$ CI } \\
\cline { 2 - 5 } Effect & Estimate & Post.SD & Lower & Upper \\
\hline DV: Log-Transformed Output Rank (Higher=More & Accessible $)$ & & & \\
Intercept & -1.18 & 0.08 & -1.35 & -1.02 \\
Hypothesized Activity RF (-0.5=Prev.; +0.5=Prom.) & -0.05 & 0.15 & -0.33 & 0.25 \\
Promotion (vs. Prevention) Strength Dominance & 0.01 & 0.06 & -0.11 & 0.13 \\
Output Total & -0.33 & 0.05 & -0.43 & -0.23 \\
Frequency of Use in English Language & 0.06 & 0.12 & -0.17 & 0.32 \\
Length & 0.05 & 0.10 & -0.14 & 0.25 \\
Activity RF×Prom. (vs. Prev.) Strength Dominance & $\mathbf{0 . 2 8}$ & $\mathbf{0 . 1 1}$ & $\mathbf{0 . 0 6}$ & $\mathbf{0 . 5 0}$ \\
\hline
\end{tabular}


Note. $\mathrm{RF}=$ Regulatory Focus; Prom.=Promotion; Prev.=Prevention. Effects for the predictor of interest are shown in bold. For the complete results of these analyses, including random effects, see Table S20 in the Supplementary Material.

\section{Discussion}

After establishing the hypothesized associations between the prevention and promotion systems and specific goal pursuit processes in Studies 1A and 1B, Study 2A provided initial evidence that the associations between regulatory focus and these goal pursuit activities predict the accessibility of these activities, operationalized as output primacy. In line with Hypothesis 3, participants' promotion ( $v s$. prevention) strength dominance interacted with the process activities' regulatory focus to predict the activities' output rank. Furthermore, the direction of this effect indicated that participants higher ( $v s$. lower) in promotion strength dominance were more likely to output promotion ( $v s$. prevention) process activities earlier in the recall task, and this effect was directionally stronger when examining only the subset containing the first 13 activities recalled by each participant.

Together with the results of Studies $1 \mathrm{~A}$ and $1 \mathrm{~B}$, these findings provide converging evidence in support of proposed framework of prevention versus promotion activities in the process of goal pursuit. However, despite the study's strengths, one limitation of Study 2A is its use of measured regulatory focus strength, which prevented us from drawing any causal inferences about the variable's impact on activity accessibility. Additionally, as noted by other scholars (e.g., Scholer et al., 2010), it is important to conduct studies that involve the measurement and the manipulation of regulatory focus, as this combined set of results are needed to provide evidence that both chronic and situationally induced regulatory focus can influence the dependent measure of interest. Finally, given that Study 2A was conducted with an online 
sample, we intended to conduct Study 2B with an in-person sample recruited from our university community to test the generalizability of these findings.

\section{Study 2B}

Study 2B aimed to replicate the findings of Study 2A within a pre-registered lab study. Importantly, Study 2B differed from Study 2A in that we experimentally manipulated participants' state regulatory focus using a well-established procedure. By using this regulatory focus strength manipulation (rather than measuring regulatory focus strength as in Study $2 \mathrm{~A}$ ), we were able to investigate whether differences in regulatory focus strength cause changes in the accessibility of motivationally relevant (vs. irrelevant) activities in the process of goal pursuit. We hypothesized that participants' assigned regulatory focus strength induction would interact with activity regulatory focus to predict the log-transformed output rank of regulatory focusspecific process activities, such that participants who completed a promotion ( $v s$. prevention) induction would tend to output promotion ( $v s$. prevention) process activities earlier during the unaided recall task.

\section{Method}

\section{Participants}

To determine the sample size for this study, we used R (Version 4.0.2; R Core Team, 2019) to conduct a Bayesian equivalent of a power analysis following a simulation-based approach (Kurz, 2019). Given that the effect of interest may be smaller than the effect found in Study 2A, our goal was to obtain power of 0.90 to detect an effect of $b=0.20$ at $\alpha=0.05$. The target sample size was 110 participants, and in order to (a) account for potential exclusions and (b) allow us to re-run all analyses among a sample of only native English speaking participants, we aimed to recruit 200 participants. We also pre-registered a stopping rule indicating that if we had collected data from at least 150 participants by the end of the Spring 2020 semester, despite 
not reaching the total sample size of 200 , we would terminate data collection and proceed to data analysis.

Data collection took place between February 2020 and March 2020. Participants were members of the university community at a university in the northeastern U.S. who had opted in to receiving recruitment materials from a behavioral research lab on campus. All participants were paid $\$ 5$. Although the pre-registration included a stopping rule of 150 participants, given the Covid-19 pandemic, data collection ended with a hiatus on human subjects research mandated by the university in March 2020. As a result, Study 2B includes data from the 125 participants who were recruited prior to this hiatus. Among this recruited sample, three participants were excluded for failing to properly complete the regulatory focus induction manipulation (i.e., the primary independent variable), leaving a sample of 122 participants. Additionally, among these participants, three participants failed to recall any activities from our framework at the end of the study (i.e., the primary dependent variable). These exclusions left a sample of 119 participants ranging in age from 18 to $68(M=24 ; S D=6)$. Participants were male $(37 \%)$ and female $(60 \%)$, with the remaining participants unspecified. They self-identified as Asian (47\%), White (24\%), multiracial (16\%), Hispanic (8\%), and Black (4\%). They indicated the highest level of formal education they have completed as a high school diploma (12\%), some college (31\%), an associate degree $(2 \%)$, a bachelor's degree $(23 \%)$, a master's degree $(27 \%)$, and a doctorate $(2 \%)$. In accordance with the requirements of the research lab in which this study took place, data on participants' household income was not collected.

Per the pre-defined exclusion criteria, we then examined the number of participants who responded that they were not native English speakers, as if this number fell below $10 \%$, we would have excluded this group. Because 44 of the remaining 119 participants $(36.1 \%)$ reported a native language other than English, these participants were not excluded from the primary 
analyses. As described in the study's pre-registration, we also conducted all analyses excluding this group and noted very similar results (see Tables S29 and S30 in the Supplementary Material). A sensitivity analysis (G*Power; Faul et al., 2009) showed that this final sample could detect an effect size of $f^{2}=0.07$ or larger for the critical interaction between Activity Regulatory Focus and participants' Regulatory Focus Induction Condition (power $=0.80 ; \alpha=0.05$; two-tailed; testing one of six total predictors).

The dataset included 1,506 total recalled activities, 916 of which were regulatory focusspecific processes from the hypothesized framework. Among the sample of participants who recalled at least one of the 24 prevention and promotion goal pursuit process activities, the number of activities recalled ranged from 1 to $16(M=7.70, S D=2.91)$. For exploratory purposes, we also conducted the primary analysis on a subset of the data including only the 110 participants who recalled at least one promotion process activity and one prevention process activity (total number of regulatory focus-specific process activities recalled among this group=897). The results of this exploratory analysis can be found in Table S28 in the Supplementary Material.

\section{Materials and Measures}

Regulatory Focus Strength Induction. We experimentally manipulated regulatory focus strength by randomly assigning participants to respond to either a prevention or promotion induction prompt. Originally developed by Higgins, Roney, Crowe, and Hymes (1994), these inductions have been used successfully in a number of studies examining the effects of regulatory focus strength on a range of different cognitive and behavioral outcomes (e.g., Freitas \& Higgins, 2002; Higgins et al., 1986; Liberman et al., 2001; Scholer et al., 2010). The induction prompts direct participants to spend five to seven minutes writing about how their current duties and obligations (prevention) versus hopes and aspirations (promotion) are different now from what they were when they were growing up. 
Activity List. The activity list used in Study 2B was identical to the list of 39 activities used in Study 1B (see Table S23 in the Supplementary Material). Importantly, it included the same 12 goal pursuit activities hypothesized to relate to prevention processes and 12 goal pursuit activities hypothesized to relate to promotion processes. Similar to Study 2 A, the regulatory focus of each of these process activities was effect-coded $(-0.5=$ prevention $;+0.5=$ promotion $)$ and manipulated on a within-participants basis in the activity importance rating task described next.

Activity Importance Rating Task. The activity importance rating task used in Study 2B was very similar to the version of the task used in Study 2A, but it contained several minor differences. Given the slightly broadened activity list in Study 2B (39 vs. 36), participants made 39 activity ratings. Further, in Study 2B, activities were rated on a nine-point Likert scale (1=not important at all to 9=absolutely essential), rather than the 101-point sliding scale. Finally, although nearly all activities were presented and rated in a random order, the activity "sleep" was rated first by all participants.

Distractor Task. In Study 2B, in order to adhere to timing and payment requirements in the research lab in which the study took place, we administered a condensed version of the distractor task used in Study 2A. This distractor task in Study 2B consisted of four patterncompletion items from Raven's Progressive Matrices (Raven \& Raven, 2003). On average, this distraction lasted 2.22 minutes.

Unaided Recall Task. In Study 2B, to measure the accessibility (i.e., output rank) of each activity, participants completed an unaided recall task nearly identical to the unaided recall task administered in Study 2A, but with 39 blank text boxes instead of 36 (to reflect the difference in the length of the activity list). As in Study 2A, research assistants hand-coded the data from this task to ensure the inclusion of any activities recalled with minor misspellings or different verb forms. For each recalled activity, coders noted the output rank of the activity, and these output 
ranks were log-transformed to normalize the distributions and multiplied by -1 so that higher scores indicated greater output primacy. Then, these output rank data were dummy-coded to produce a binary measure of output $(0=$ not recalled; $1=$ recalled $)$, and an output total score was calculated by summing the number of activities that each participant recalled and rescaling these sums $(M=0 ; S D=1)$.

Further Measures. As in Studies 1A and 1B, to control for any potential effects of stimulus length or frequency, we measured each of these variables for all activities. Additionally, we measured participants' prevention pride $(M=3.36 ; S D=0.85 ; \alpha=0.78)$ and promotion pride $(M=3.53 ; S D=0.64 ; \alpha=0.68)$ scores using the RFQ (Higgins et al., 2001), and their assessment $(M=4.23 ; S D=0.63 ; \alpha=0.74)$ and locomotion $(M=4.41 ; S D=0.59 ; \alpha=0.76)$ orientations using the RMQ (Kruglanski et al., 2000).

\section{Procedure}

This study involved an experimental lab design containing two purportedly separate studies. The purpose of this "multiple-study paradigm" design was to prevent participants' responses to tasks they completed in the first part of the study from biasing their performance in later tasks. Upon providing consent, participants learned that they would begin a purported Study 1 investigating how people's standards change over time. Using complete random assignment, each participant was assigned to one of the two between-subjects regulatory focus induction conditions (prevention vs. promotion). After completing this induction, participants were directed to a purported Study 2 on the topic of motivation and problem solving. Participants then proceeded to the activity importance rating task, thus encountering all 39 activities for the first time. Then, participants completed the brief distractor task, which was followed by the unaided recall task probing the output primacy of the various activities. After completing these tasks, participants completed two exploratory motivational measures: the RMQ and the RFQ. Finally, 
participants optionally provided brief demographic information and were debriefed, thanked, and compensated.

\section{Results}

\section{Analytic Strategy}

As in Study 2A, and in line with Hypothesis 3, we predicted that the accessibility of prevention versus promotion process activities, operationalized as output rank, would be associated with participants' regulatory focus strength. However, one key difference in Study 2B was that regulatory focus strength was manipulated, rather than measured. Thus, the primary analysis examined whether the interaction between the hypothesized regulatory focus of a given process activity and each participant's experimentally induced regulatory focus strength predicted the log-transformed output rank of the activity. Like our analytic approach in Studies 1A and 1B, we selected the subset of the data comprising only activities we hypothesized to reflect prevention- or promotion-specific processes $(N=916)$ and analyzed these data within a Bayesian multilevel regression analysis. This model was specified identically to the model used in Study 2A's primary analysis, with the exception of participants' promotion ( $v s$. prevention) strength dominance; in Study 2B, this variable was replaced with an effect-coded variable reflecting participants' regulatory focus induction condition $(-0.5=$ prevention,$+0.5=$ promotion $)$.

Additionally, as in Study 2A, we were particularly interested in how output rank (i.e., a measure of output primacy) was influenced by the interaction between induced regulatory focus strength and the hypothesized regulatory focus, beyond any differences in output total. These initial analyses revealed two sets of correlations between participants' output total and two variables included in the primary analyses, including participants' effect-coded regulatory focus induction condition $(r=0.16$; 95\% CI: $-0.01,0.33)$ and participants' average log-transformed output rank for activities from the hypothesized framework $(r=-0.78 ; 95 \% \mathrm{CI}:-0.84,-0.70)$. 
Given these correlations, like in Study 2A, we conducted a "leave-one-out" cross-validation analysis comparing models including versus excluding output total, and this analysis indicated that the model with this covariate had greater expected predictive accuracy. Thus, as in Study 2A, the results of this revised model are included below. (All results using the original pre-registered model without output total included as a covariate are provided in and following Table S26 in the Supplementary Material.) For descriptive statistics and correlations among all variables included within these models, see Table S24 in the Supplementary Material.

Further, because we hypothesized that accessible activities would be recalled earliest in the memory task, as in Study 2A, we also conducted the primary analysis with a subset of the data containing only the first 13 activities that participants recalled within the unaided recall task, because these earlier ( $v s$. later) recalled activities are more likely to reflect output primacy. As a result, we report the results of both our analyses conducted the full dataset as well as the results of analyses that included only the regulatory focus-specific process activities included in participants' first 13 recalled activities below. Additionally, we conducted an exploratory exact binomial test of goodness-of-fit to examine differences in the accessibility (i.e., output rank) of prevention versus promotion process activities between the two induction conditions. Although these exploratory analyses were not pre-registered, we provide the results of these investigations below.

Finally, although the primary analysis contains data for all participants, given that these analyses differentiate between the average output rank for promotion versus prevention process activities, we also conducted these analyses only including data for participants who remembered at least one promotion activity and at least one prevention activity. The results of these analyses are presented in Table S28 in the Supplementary Material.

\section{Output Total: Number of Activities Recalled}


On average, participants recalled 12.66 total activities $(S D=4.23)$ in the unaided recall task. Among these recalled activities, participants recalled an average of 7.70 activities that we hypothesized were associated with prevention or promotion processes $(S D=2.91)$, comprising an average of 3.83 prevention activities $(S D=1.70)$ and 3.87 promotion activities $(S D=1.88)$. However, by estimating the difference between the number of promotion versus prevention process activities recalled within a Bayesian linear regression model, we determined that this difference did not differ from zero (95\% CI: $-0.33,0.42)$.

\section{Output Rank: Primacy of Activities Recalled}

Across participants, the average log-transformed output rank among the recalled activities in the unaided recall task was $-1.81(S D=0.79)$. In contrast to Study $2 \mathrm{~A}$, promotion process activities $(M=-1.80 ; S D=0.80)$ tended to be recalled slightly earlier than prevention process activities $(M=-1.89 ; S D=0.74)$. However, by estimating the difference between log-transformed output rank promotion versus prevention process activities within a Bayesian linear regression model, we determined that this difference did not differ from zero (95\% CI: $-0.002,0.23)$. Primary Analysis: Does the Interaction Between Participants' Induced Regulatory Focus Strength and Activity Regulatory Focus Predict the Output Rank of Activities in the

\section{Framework?}

We hypothesized that the interaction between participants' promotion (vs. prevention) strength dominance and the regulatory focus of each prevention- or promotion-specific process activity in the hypothesized framework would predict that activity's log-transformed output rank. To investigate this question, we examined the model output from the Bayesian multilevel regression analysis (see Table 7). The effect size for this interaction was 0.09 units, with a $95 \%$ credible interval that included zero $(-0.10,0.28)$. Based on this credible interval, contrary to Hypothesis 3, these results did not indicate that the interaction between these variables influences 
regulatory focus-specific process activities' output rank. Additionally, this analysis indicated that output total was the only predictor of log-transformed output rank $(b=-0.28 ; 95 \% \mathrm{CI}$ : $-0.34,-$ $0.23)$.

\section{Table 7}

Parameter Estimates for Multilevel Multiple Regression Analysis Predicting Log-Transformed Output Rank of Regulatory Focus-Specific Process Activities (Study 2B; Fixed Effects Only)

\begin{tabular}{|c|c|c|c|c|}
\hline \multirow[b]{2}{*}{ Effect } & \multirow[b]{2}{*}{ Estimate } & \multirow[b]{2}{*}{ Post.SD } & \multicolumn{2}{|c|}{$95 \% \mathrm{CI}$} \\
\hline & & & Lower & Upper \\
\hline \multicolumn{5}{|c|}{ DV: Log-Transformed Output Rank (Higher=More Accessible) } \\
\hline Intercept & -1.76 & 0.03 & -1.83 & -1.69 \\
\hline Hypothesized Activity RF $(-0.5=$ Prev.; $+0.5=$ Prom.$)$ & 0.08 & 0.07 & -0.05 & 0.21 \\
\hline RF Induction Condition $(-0.5=$ Prev.; $+0.5=$ Prom.$)$ & 0.01 & 0.05 & -0.08 & 0.11 \\
\hline Output Total & -0.28 & 0.03 & -0.34 & -0.23 \\
\hline Frequency of Use in English Language & -0.01 & 0.05 & -0.10 & 0.09 \\
\hline Length & 0.02 & 0.04 & -0.07 & 0.11 \\
\hline Activity RF $\times$ RF Induction Condition & 0.09 & 0.10 & $-\mathbf{0 . 1 0}$ & 0.28 \\
\hline
\end{tabular}

Note. RF=Regulatory Focus; Prom.=Promotion; Prev.=Prevention. Effects for the predictor of

interest are shown in bold. For the complete results of this analysis, including random effects, see Table S25 in the Supplementary Material.

Exploratory Analysis: When Examining Activities with the Greatest Output Primacy, Does the Interaction Between Participants' Induced Regulatory Focus Strength and Activity Regulatory Focus Predict the Output Rank of Activities in the Framework?

Like in Study 2A, we also conducted an exploratory analysis with a subset of the data containing only the first 13 activities recalled by participants. The model output from this Bayesian multilevel regression analysis indicated an effect size for the interaction of interest of 0.20 units, with a $95 \%$ credible interval that did not cross zero $(0.002,0.39$; see Table 8 and Figure 6). Based on this credible interval and the positive direction of the effect, these results supported Hypothesis 3, suggesting that the interaction between these variables influenced the 
log-transformed output rank of prevention- and promotion-specific goal pursuit process activities when examining only participants' first 13 recalled activities.

\section{Table 8}

Parameter Estimates for Multilevel Multiple Regression Analysis Predicting Log-Transformed Output Rank of Regulatory Focus-Specific Process Activities (Study 2B; Data from First 13 Outputted Activities Only; Fixed Effects Only)

\begin{tabular}{lrrrr}
\hline & & & \multicolumn{2}{c}{$95 \%$ CI } \\
\cline { 4 - 5 } Effect & Estimate & Post.SD & Lower & Upper \\
\hline DV: Log-Transformed Output Rank (Higher=More & Accessible $)$ & & & \\
Intercept & -1.66 & 0.04 & -1.73 & -1.59 \\
Hypothesized Activity RF (-0.5=Prev.; +0.5=Prom.) & 0.07 & 0.07 & -0.07 & 0.21 \\
RF Induction Condition (-0.5=Prev.; +0.5=Prom.) & 0.04 & 0.05 & -0.06 & 0.13 \\
Output Total & -0.13 & 0.03 & -0.19 & -0.07 \\
Frequency of Use in English Language & -0.01 & 0.05 & -0.11 & 0.10 \\
Length & 0.01 & 0.05 & -0.08 & 0.11 \\
Activity RF×RF Induction Condition & $\mathbf{0 . 2 0}$ & $\mathbf{0 . 1 0}$ & $\mathbf{0 . 0 0 2}$ & $\mathbf{0 . 3 9}$ \\
\hline
\end{tabular}

Note. RF=Regulatory Focus; Prom.=Promotion; Prev.=Prevention. Effects for the predictor of interest are shown in bold. For the complete results of these analyses, including random effects, see Table S27 in the Supplementary Material.

\section{Figure 6}

Log-Transformed Output Rankfor Promotion Versus Prevention Process Activities as a Function of Promotion Strength Dominance (Study 2B; Data from First 13 Outputted Activities Only) 


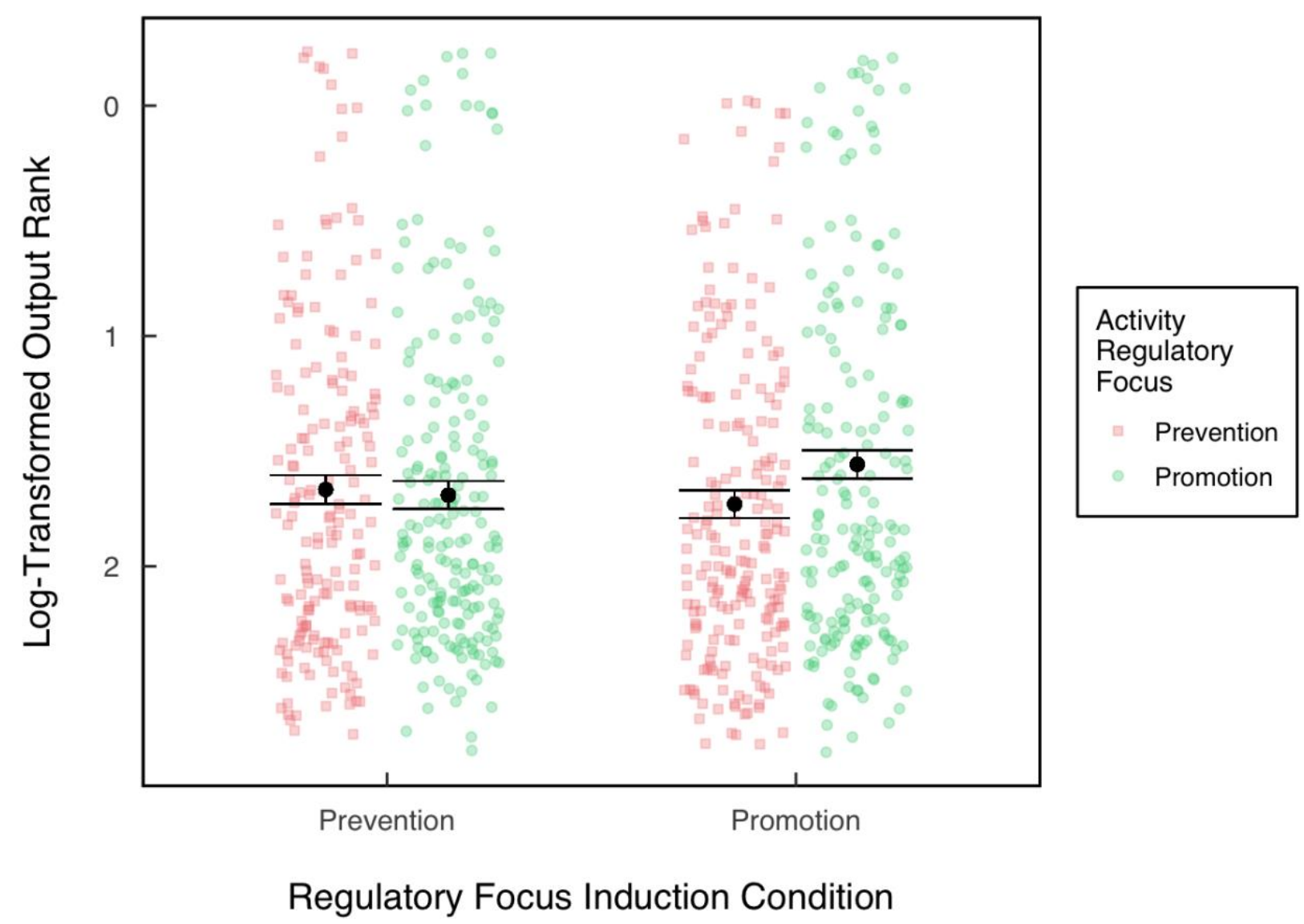

Note. Model-predicted log-transformed output rank for promotion versus prevention process activities as a function of participants' regulatory focus induction condition, at typical levels of output total, activity frequency of use in the English language, and activity length. The logtransformed output rank values on the $\mathrm{Y}$-axis have been reversed so that output ranks higher in the plot indicate greater accessibility. The red (dark gray) squares represent outputted prevention process activities. The green (light gray) circles represent outputted promotion process activities. Error bars represent standard errors.

\section{Exploratory Analysis: Binomial Exact Test}

In addition to the Bayesian regression analyses described above, we also conducted an exploratory binomial exact test of goodness-of-fit to compare on an activity-specific basis the log-transformed output rank of prevention versus promotion process activities among participants 
who completed a prevention versus promotion strength induction. Beginning with the subset of data comprising participants' first 13 recalled process activities in the recall task, we calculated the mean log-transformed output rank for each regulatory focus-specific activity in the prevention condition and the promotion condition. Next, for each of these activities, we calculated an activity-specific promotion ( $v s$. prevention) dominance score in terms of log-transformed output rank by subtracting the mean in the prevention condition from the mean in the promotion condition. Then, we compared each of the promotion process activities with each of the prevention process activities based upon these activity-specific output rank promotion-dominance scores (see Figure 7 for a visualization of these scores). Given that the dataset included such scores for 12 promotion activities and 12 prevention activities, we compared these scores for 144 activity pairs. In 109 of these 144 activity pairs, the promotion activity had an output rank score higher in promotion-dominance than the prevention activity (in line with Hypothesis 3). Importantly, if these activities did not differ in their relevance to the promotion versus prevention systems, we would not have expected the proportion of activity pairings in line with our hypotheses to differ from chance (i.e., 72 of the 144 pairings). Based on a two-sided binomial test, these observed values differ significantly from the "chance" proportion, $p<.0001$.

\section{Figure 7}

Difference in Log-Transformed Output Rank of Regulatory Focus-Specific Process Activities in the Promotion Versus Prevention Strength Induction Conditions (Study 2B; Data from First 13 


\section{Outputted Activities Only)}

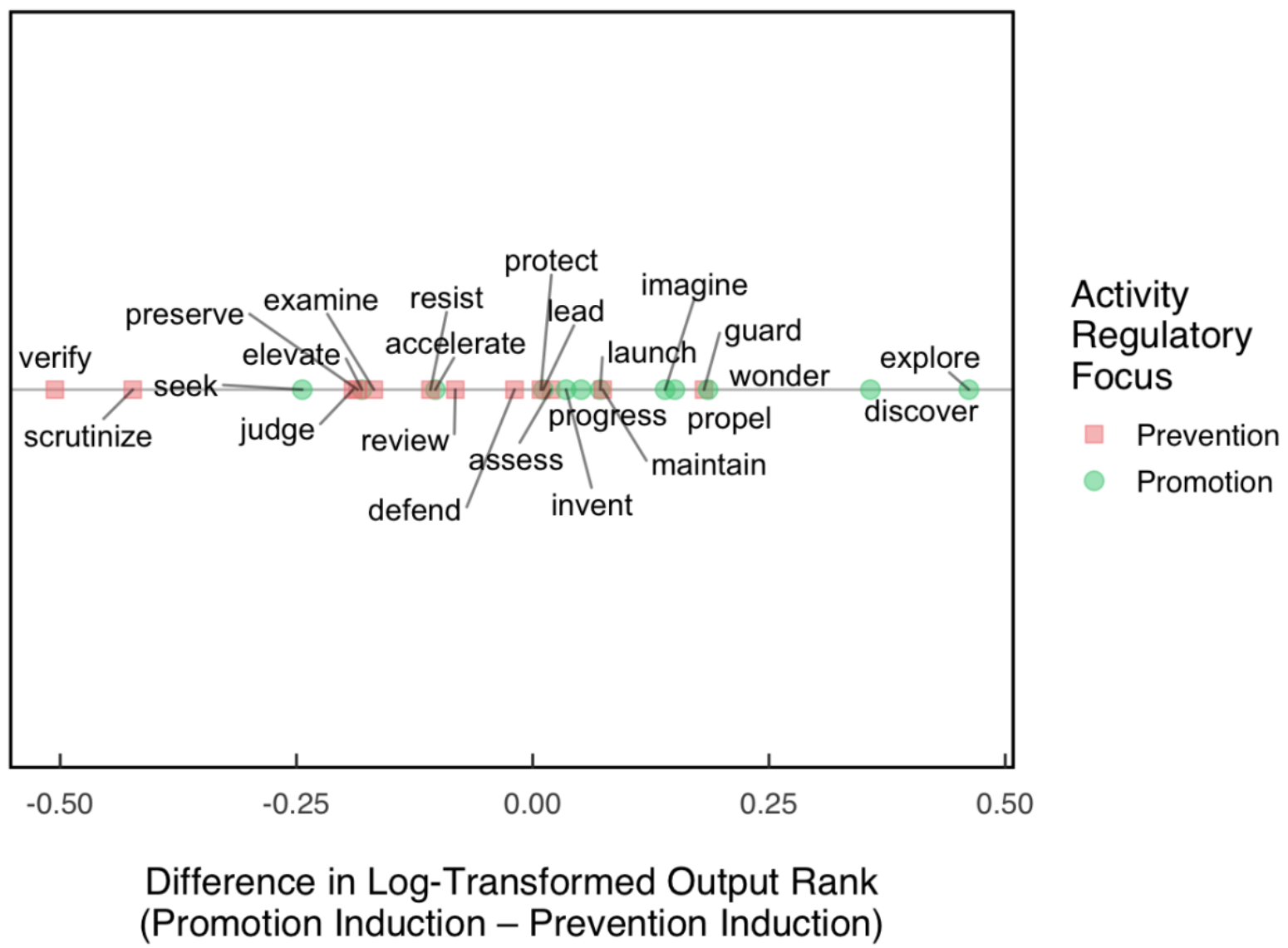

Note. Mean difference in log-transformed output rank for each regulatory focus-specific process activity among participants in the promotion versus prevention strength induction conditions, when calculated based on the subset of data containing participants' first 13 recalled activities. The red (dark gray) squares represent prevention process activities. The green (light gray) circles represent promotion process activities. On average, activities with values greater than zero were more accessible following a promotion (vs. prevention) induction; activities with values less than zero were more accessible following a prevention (vs. promotion) induction.

\section{Discussion}

Study 2B provided some additional evidence for Hypothesis 3, revealing that the interaction between regulatory focus strength and activity regulatory focus produced differences 
in the accessibility of regulatory focus-specific process activities when specifically examining the 13 activities outputted first in the unaided recall task. This evidence from Bayesian regression analyses similar to those conducted in Study 2A was complemented by converging evidence from the exploratory binomial exact test.

Interestingly, when the primary analysis was conducted with the log-transformed output rank data outputted at any point in the unaided recall task ( $v s$. the first 13 recalled activities), the size of the interaction effect of interest was approximately halved and the $95 \%$ credible interval for interaction effect shifted to include zero. This finding may illuminate an important boundary condition of the effects highlighted in the present research: While inducing a promotion or prevention focus does appear to influence the accessibility of relevant process activities, the extent of this effect may depend on output primacy. Additionally, although we found a slight increase in the size of the key interaction effect in Study 2A when examining only the first 13 recalled activities, this difference was far larger in Study 2B. This distinction may relate to the fact that participants' regulatory focus in Study 2A was determined using a measure of their chronic regulatory focus, whereas in Study 2B, we created a situational change in regulatory focus using an experimental manipulation. In practical terms, these minor differences in the results of Studies $2 \mathrm{~A}$ and $2 \mathrm{~B}$ suggest that although a given situation may prime a prevention or promotion focus, and this state regulatory focus may influence the relevance of different goal pursuit activities, this increase in motivational relevance may not be maintained indefinitely. In contrast, individual differences in the dominance of people's regulatory focus strength may be relatively more stable. Together with the results of Study $2 \mathrm{~A}$, these findings provided converging experimental support for our hypothesized framework. 


\section{General Discussion}

Although the prevention and promotion systems posited within regulatory focus theory are known to be associated with distinct goal pursuit activities, investigations into these associations have seemingly lacked a comprehensive guiding framework. To the best of our knowledge, the present research is the first to put forth such a framework, which details the different kinds of truth- and control-oriented goal pursuit process activities motivated by prevention and promotion. By combining regulatory focus theory, which traditionally has been conceptualized in the domain of value, with truth and control, this novel framework integrates distinct theories of motivation that have not previously been studied together. In doing so, it highlights the dynamic interplay of various motivational systems that drive human behavior.

Across four studies using both experimental and correlational methods, results indicated that the associations between (a) the prevention and promotion systems and (b) regulatory focusspecific goal pursuit process activities are broadly available, highly accessible, and interact with individual differences in regulatory focus strength to predict the activities' motivational relevance. This work contributes to the motivation and self-regulation literatures by extending the distinction between the prevention system's preference for vigilance and the promotion system's preference for eagerness (Crowe \& Higgins, 1997; Higgins et al., 1994) to propose how these general tendencies influence the motivational relevance of specific activities involved in the process of goal pursuit. It integrates past regulatory focus research examining system-specific preferences related to topics as wide-ranging as curiosity, political ideology, discounting under uncertainty, and leadership styles. Given that these preferences relate to areas of psychology spanning cognition, judgment and decision making, and social influence, it is our hope that this framework will be useful and generative in suggesting opportunities for future research to scholars from a wide range of disciplines. 


\section{Limitations and Future Directions}

Despite its strengths, the present research has its limitations. Most notably, although we found that individual differences in participants' regulatory focus strength predicted the accessibility of process activities relevant to their predominant regulatory focus in Study $2 \mathrm{~A}$, when experimentally manipulating participants' state regulatory focus in Study 2B, we only found evidence for the hypothesized effect when examining a smaller group of activities outputted earliest by these participants. It is possible that differences in the samples' engagement in the studies produced this difference in the effect. As evidence for this explanation, the M-Turk participants who recalled at least one activity in Study 2A outputted 6.98 activities on average, whereas the university participants who recalled at least one activity in Study 2B outputted an average of 12.66 activities. It may be the case that the interaction between participants' regulatory focus (measured or manipulated) and activities' regulatory focus only affect initial output (vs. an entire stream of output), but that we were not able to detect this difference among the M-Turk sample because the number of activities participants outputted simply did not reach this threshold. However, an alternative explanation is also possible. It may be the case that the activation of the regulatory focus induced by the priming in Study $2 \mathrm{~B}$ decreased throughout the course of the study, thus impacting more strongly the activities that were outputted earlier ( $v s$. later). Further research is required to determine if either of these possible explanations is correct. Importantly, if follow-up research indicates that the priming does, in fact, wear off during the course of the study, future work should be done to confirm this shift in accessibility and pinpoint its time course. Understanding the duration during which such an induction is effective would be particularly important for researchers interested in developing interventions to promote specific types of goal pursuit processes. 
The present research also offers broader avenues for future work. With the proposed framework as a guide, researchers can explore how the interactions of these systems relate to other motivational constructs. For instance, this research suggests that the promotion and prevention systems may motivate distinct truth-seeking activities in the process of goal pursuit. Given that promotion-focused truth-oriented activities reflect the generation and growth of knowledge (e.g., explore, discover), we would predict that a promotion focus will be uniquely associated with trait measures of epistemic curiosity, particularly subscales related to joyous exploration (Kashdan et al., 2018) and interest (Litman \& Spielberger, 2003). In contrast, given that prevention-focused truth-oriented activities reflect a desire for certainty and error minimization (e.g., judge, verify), we would predict that a prevention focus will be uniquely associated with the need for closure (in particular, preferences for order and predictability, as well as discomfort with ambiguity; Kruglanski et al., 1993; Webster \& Kruglanski, 1994). Such work is important because these distinct truth-oriented activities are critical to an effective goal pursuit process, yet must be deployed strategically; for example, knowledge refinement is often only useful once enough knowledge has been gathered first through curious exploration.

Finally, this framework has important implications for the study of goal-directed behavior and performance, and offers an approach to begin filling critical gaps in the field's knowledge of goal pursuit processes. This proposal is grounded in Eitam and Higgins' (2010) relevance of a representation framework, which posits that motivationally relevant constructs influence cognition and behavior. If the prevention versus promotion systems systematically motivate distinct goal pursuit process activities as suggested by the present research, future studies should examine these proposed behavioral implications explicitly. We would hypothesize that: (a) these associations between regulatory focus and goal pursuit activities will be reflected in actual behavioral choices, and (b) present concern with each of these four motive domains will enhance 
performance on activities in that domain. To test these hypotheses experimentally, researchers could manipulate participants' current motivational concerns by creating writing inductions specific to each activity domain and then measure task choice and performance. By going beyond accessibility measures to investigate how the interactions of these motives affect behavior, this future research will provide valuable insight on why and how the process of goal pursuit varies across individuals and situations. Furthermore, if such work indicates that these motive inductions effectively influence behavior and performance, these results would suggest how, through related real-world interventions, people might engage in more productive and enjoyable goal pursuit journeys. For instance, scholars might apply this knowledge to determine how individuals, managers, and educators can strategically promote the specific kinds of truth and control activities befitting the goals of themselves, their teams, or their students. Given that both economic success and well-being relate to how effectively people pursue their goals (Ameriks et al., 2003; Klug \& Maier, 2015), we suggest that this is a worthy aim.

\section{Open Practices}

Pre-registrations for all replication studies can be found on the Open Science Framework (OSF; Study 1B: https://osf.io/xh7qk/?view_only=19651dfde9c148ef928544d4292710f8; Study 2B: https://osf.io/efj9q/?view_only=de3a9c0121854e9c8eda03afb4b5894c). Further, the OSF project page associated with the present research (https://osf.io/ybcaq/?view_only=8bf7abc0419f4f618662f6c870aaea60) contains the research materials, data cleaning code, analysis code, and cleaned, de-identified data associated with all studies. 


\section{References}

Ameriks, J., Caplin, A., \& Leahy, J. (2003). Wealth accumulation and the propensity to plan. The Quarterly Journal of Economics, 118(3), 1007-1047. https://doi.org/10.1162/00335530360698487

Atkinson, J. W. (1957). Motivational determinants of risk-taking behavior. Psychological Review, 64(6), 359-372. https://doi.org/10.1037/h0043445

Avnet, T., \& Higgins, E. T. (2006). How regulatory fit affects value in consumer choices and opinions. Journal of Marketing Research, 43(1), 1-10. https://doi.org/10.1509/jmkr.43.1.1

Bentham, J. (1823). An introduction to the principles of morals and legislation (2nd ed., Vol. 1). W. Pickering.

Beuk, F., \& Basadur, T. (2016). Regulatory focus, task engagement and divergent thinking. Creativity \& Innovation Management, 25(2), 199-210. https://doi.org/10.1111/caim.12182

Boldero, J. M., \& Higgins, E. T. (2011). Regulatory focus and political decision making: When people favor reform over the status quo. Political Psychology, 32(3), 399-418. https://doi.org/10.1111/j.1467-9221.2010.00814.x

Bürkner, P.-C. (2017). brms: An R package for Bayesian multilevel models using Stan. Journal of Statistical Software, 80(1), 1-28. https://doi.org/10.18637/jss.v080.i01

Bürkner, P.-C. (2018). Advanced Bayesian multilevel modeling with the R package brms. The $R$ Journal, 10(1), 395-411. https://doi.org/10.32614/RJ-2018-017

Carpenter, T. P., Pogacar, R., Pullig, C., Kouril, M., Aguilar, S., LaBouff, J., Isenberg, N., \& Chakroff, A. (2019). Survey-software implicit association tests: A methodological and 
empirical analysis. Behavior Research Methods, 51(5), 2194-2208.

https://doi.org/10.3758/s13428-019-01293-3

Carver, C. S., \& Scheier, M. F. (1998). On the self-regulation of behavior. Cambridge University Press.

Cohen, J. (1988). Statistical power analysis for the behavioral sciences (2nd ed.). Lawrence Erlbaum Associates.

Cornwell, J. F. M., \& Higgins, E. T. (2013). Morality and its relation to political ideology: The role of promotion and prevention concerns. Personality and Social Psychology Bulletin, 39(9), 1164-1172. https://doi.org/10.1177/0146167213489036

Cornwell, J. F. M., \& Higgins, E. T. (2016). Eager feelings and vigilant reasons: Regulatory focus differences in judging moral wrongs. Journal of Experimental Psychology: General, 145(3), 338-355. https://doi.org/10.1037/xge0000136

Crowe, E., \& Higgins, E. T. (1997). Regulatory focus and strategic inclinations: Promotion and prevention in decision-making. Organizational Behavior and Human Decision Processes, 69(2), 117-132. https://doi.org/10.1006/obhd.1996.2675

Davies, M. (2008). The Corpus of Contemporary American English (COCA): One billion million words, 1990-2019. https://www.english-corpora.org/coca/

Eitam, B., \& Higgins, E. T. (2010). Motivation in mental accessibility: Relevance of a representation (ROAR) as a new framework. Social and Personality Psychology Compass, 4(10), 951-967. https://doi.org/10.1111/j.1751-9004.2010.00309.x

Eitam, B., \& Higgins, E. T. (2014). What's in a goal? The role of motivational relevance in cognition and action. Behavioral and Brain Sciences, 37(2), 141-142. https://doi.org/10.1017/S0140525X13002008 
Eitam, B., Miele, D. B., \& Higgins, E. T. (2013). Motivated remembering: Remembering as accessibility and accessibility as motivational relevance. In D. Carlston (Ed.), The Oxford handbook of social cognition (pp. 463-475). Oxford University Press.

Faul, F., Erdfelder, E., Buchner, A., \& Lang, A.-G. (2009). Statistical power analyses using G*Power 3.1: Tests for correlation and regression analyses. Behavior Research Methods, 41(4), 1149-1160. https://doi.org/10.3758/BRM.41.4.1149

Förster, J., Higgins, E. T., \& Bianco, A. T. (2003). Speed/accuracy decisions in task performance: Built-in trade-off or separate strategic concerns? Organizational Behavior and Human Decision Processes, 90(1), 148-164. https://doi.org/10.1016/S0749-5978(02)00509-5

Freitas, A. L., \& Higgins, E. T. (2002). Enjoying goal-directed action: The role of regulatory fit. Psychological Science, 13(1), 1-6. https://doi.org/10.1111/1467-9280.00401

Friedman, R. S., \& Förster, J. (2005). Effects of motivational cues on perceptual asymmetry: Implications for creativity and analytical problem solving. Journal of Personality and Social Psychology, 88(2), 263-275. https://doi.org/10.1037/0022-3514.88.2.263

Gamache, D. L., McNamara, G., Mannor, M. J., \& Johnson, R. E. (2015). Motivated to acquire? The impact of CEO regulatory focus on firm acquisitions. Academy of Management Journal, 58(4), 1261-1282. https://doi.org/10.5465/amj.2013.0377

Gollwitzer, P. M. (1990). Action phases and mind-sets. In E. T. Higgins \& R. M. Sorrentino (Eds.), Handbook of motivation and cognition: Foundations of social behavior (Vol. 2, pp. 53-92). Guilford Press.

Halamish, V., Liberman, N., Higgins, E. T., \& Idson, L. C. (2008). Regulatory focus effects on discounting over uncertainty for losses vs. Gains. Journal of Economic Psychology, 29(5), 654-666. https://doi.org/10.1016/j.joep.2007.09.002 
Higgins, E. T. (1996). Knowledge activation: Accessibility, applicability, and salience. In E. T. Higgins \& A. W. Kruglanski (Eds.), Social psychology: Handbook of basic principles (pp. 133-168). Guilford Press.

Higgins, E. T. (1997). Beyond pleasure and pain. American Psychologist, 52(12), 1280-1300. https://doi.org/10.1037/0003-066X.52.12.1280

Higgins, E. T. (1998). Promotion and prevention: Regulatory focus as a motivational principle. In M. P. Zanna (Ed.), Advances in experimental social psychology (Vol. 30, pp. 1-46). Academic Press. https://doi.org/10.1016/S0065-2601(08)60381-0

Higgins, E. T. (2000). Making a good decision: Value from fit. American Psychologist, 55(11), 1217-1230. https://doi.org/10.1037/0003-066X.55.11.1217

Higgins, E. T. (2012). Beyond pleasure and pain: How motivation works. Oxford University Press.

Higgins, E. T. (2018). What distinguishes promotion and prevention? Attaining " +1 " from "0" as non-gain versus maintaining “0” as non-loss. Polish Psychological Bulletin, 49(1), 40-49. https://doi.org/10.24425/119470

Higgins, E. T., Bond, R. N., Klein, R., \& Strauman, T. (1986). Self-discrepancies and emotional vulnerability: How magnitude, accessibility, and type of discrepancy influence affect. Journal of Personality and Social Psychology, 51(1), 5-15. https://doi.org/10.1037/00223514.51 .1 .5

Higgins, E. T., Cesario, J., Hagiwara, N., Spiegel, S., \& Pittman, T. (2010). Increasing or decreasing interest in activities: The role of regulatory fit. Journal of Personality and Social Psychology, 98(4), 559-572. https://doi.org/10.1037/a0018833

Higgins, E. T., Friedman, R., Harlow, R. E., Idson, L. C., Ayduk, O. N., \& Taylor, A. (2001). Achievement orientations from subjective histories of success: Promotion pride versus 
prevention pride. European Journal of Social Psychology, 31(1), 3-23.

https://doi.org/10.1002/ejsp.27

Higgins, E. T., Kruglanski, A. W., \& Pierro, A. (2003). Regulatory mode: Locomotion and assessment as distinct orientations. In M. P. Zanna (Ed.), Advances in experimental social psychology (Vol. 35, pp. 293-344). Academic Press. https://doi.org/10.1016/S00652601(03)01005-0

Higgins, E. T., Pierro, A., \& Kruglanski, A. W. (2008). Re-thinking culture and personality: How self-regulatory universals create cross-cultural differences. In R. M. Sorrentino \& S. Yamaguchi (Eds.), Handbook of motivation and cognition across cultures (pp. 161-190). Academic Press. https://doi.org/10.1016/B978-0-12-373694-9.00008-8

Higgins, E. T., Roney, C. J. R., Crowe, E., \& Hymes, C. (1994). Ideal versus ought predilections for approach and avoidance: Distinct self-regulatory systems. Journal of Personality and Social Psychology, 66(2), 276-286. https://doi.org/10.1037/0022-3514.66.2.276

Higgins, E. T., Shah, J., \& Friedman, R. (1997). Emotional responses to goal attainment: Strength of regulatory focus as moderator. Journal of Personality and Social Psychology, 72(3), 515-525. https://doi.org/10.1037/0022-3514.72.3.515

Huang, J. Y., \& Bargh, J. A. (2014). The selfish goal: Autonomously operating motivational structures as the proximate cause of human judgment and behavior. Behavioral and Brain Sciences, 37(2), 121-135. https://doi.org/10.1017/S0140525X13000290

Hull, C. L. (1950). Behavior postulates and corollaries. Psychological Review, 57(3), 173-180. https://doi.org/10.1037/h0062809

Kanze, D., Conley, M. A., \& Higgins, E. T. (2019). The motivation of mission statements: How regulatory mode influences workplace discrimination. Organizational Behavior and 
Human Decision Processes, Advance online publication.

https://doi.org/10.1016/j.obhdp.2019.04.002

Kark, R., \& Van Dijk, D. (2007). Motivation to lead, motivation to follow: The role of the selfregulatory focus in leadership processes. The Academy of Management Review, 32(2), 500-528. JSTOR. https://doi.org/10.2307/20159313

Kashdan, T. B., Stiksma, M. C., Disabato, D. J., McKnight, P. E., Bekier, J., Kaji, J., \& Lazarus, R. (2018). The five-dimensional curiosity scale: Capturing the bandwidth of curiosity and identifying four unique subgroups of curious people. Journal of Research in Personality, 73, 130-149. https://doi.org/10.1016/j.jrp.2017.11.011

Kennedy, R., Clifford, S., Burleigh, T., Jewell, R., \& Waggoner, P. (2018). The shape of and solutions to the MTurk quality crisis (SSRN Scholarly Paper ID 3272468). Social Science Research Network. https://papers.ssrn.com/abstract=3272468

Kennedy, R., \& Waggoner, P. (2019). RIP: Detects fraud in online surveys by tracing, scoring, and visualizing IP addresses. https://CRAN.R-project.org/package=rIP

Klug, H. J. P., \& Maier, G. W. (2015). Linking goal progress and subjective well-being: A metaanalysis. Journal of Happiness Studies, 16(1), 37-65. https://doi.org/10.1007/s10902-0139493-0

Kruglanski, A. W., Thompson, E. P., Higgins, E. T., Atash, M. N., Pierro, A., Shah, J. Y., \& Spiegel, S. (2000). To "do the right thing" or to "just do it": Locomotion and assessment as distinct self-regulatory imperatives. Journal of Personality and Social Psychology, 79(5), 793-815. https://doi.org/10.1037/0022-3514.79.5.793

Kruglanski, A. W., Webster, D. M., \& Klem, A. (1993). Motivated resistance and openness to persuasion in the presence or absence of prior information. Journal of Personality and Social Psychology, 65(5), 861-876. https://doi.org/10.1037/0022-3514.65.5.861 
Kruschke, J. K. (2011). Bayesian assessment of null values via parameter estimation and model comparison. Perspectives on Psychological Science, 6(3), 299-312. https://doi.org/10.1177/1745691611406925

Kurz, A. S. (2019, July 18). Bayesian power analysis: Part I. Prepare to reject HO with simulation [Blog post]. A. Solomon Kurz. https://solomonkurz.netlify.app/post/bayesianpower-analysis-part-i/

Lee, A. Y., Keller, P. A., \& Sternthal, B. (2010). Value from regulatory construal fit: The persuasive impact of fit between consumer goals and message concreteness. Journal of Consumer Research, 36(5), 735-747. https://doi.org/10.1086/605591

Liberman, N., Idson, L. C., Camacho, C. J., \& Higgins, E. T. (1999). Promotion and prevention choices between stability and change. Journal of Personality and Social Psychology, 77(6), 1135-1145. https://doi.org/10.1037/0022-3514.77.6.1135

Liberman, N., Molden, D. C., Idson, L. C., \& Higgins, E. T. (2001). Promotion and prevention focus on alternative hypotheses: Implications for attributional functions. Journal of Personality and Social Psychology, 80(1), 5-18. https://doi.org/10.1037/0022-3514.80.1.5

Litman, J. A., \& Spielberger, C. D. (2003). Measuring epistemic curiosity and its diversive and specific components. Journal of Personality Assessment, 80(1), 75-86. https://doi.org/10.1207/S15327752JPA8001_16

Memmert, D., Hüttermann, S., \& Orliczek, J. (2013). Decide like Lionel Messi! The impact of regulatory focus on divergent thinking in sports. Journal of Applied Social Psychology, 43(10), 2163-2167. https://doi.org/10.1111/jasp.12159

Plessner, H., Unkelbach, C., Memmert, D., Baltes, A., \& Kolb, A. (2009). Regulatory fit as a determinant of sport performance: How to succeed in a soccer penalty-shooting. 
Psychology of Sport and Exercise, 10(1), 108-115.

https://doi.org/10.1016/j.psychsport.2008.02.001

R Core Team. (2019). R: A language and environment for statistical computing. https://www.Rproject.org/

Raven, J., \& Raven, J. (2003). Raven Progressive Matrices. In R. S. McCallum (Ed.), Handbook of nonverbal assessment (pp. 223-237). Kluwer Academic / Plenum Publishers. https://doi.org/10.1007/978-1-4615-0153-4_11

Scholer, A. A., Zou, X., Fujita, K., Stroessner, S. J., \& Higgins, E. T. (2010). When risk seeking becomes a motivational necessity. Journal of Personality and Social Psychology, 99(2), 215-231. https://doi.org/10.1037/a0019715

Vehtari, A., Gelman, A., \& Gabry, J. (2017). Practical Bayesian model evaluation using leaveone-out cross-validation and WAIC. Statistics and Computing, 27(5), 1413-1432. https://doi.org/10.1007/s11222-016-9696-4

Wallace, J. C., Butts, M. M., Johnson, P. D., Stevens, F. G., \& Smith, M. B. (2016). A multilevel model of employee innovation: Understanding the effects of regulatory focus, thriving, and employee involvement climate. Journal of Management, 42(4), 982-1004. https://doi.org/10.1177/0149206313506462

Webster, D. M., \& Kruglanski, A. W. (1994). Individual differences in need for cognitive closure. Journal of Personality and Social Psychology, 67(6), 1049-1062. https://doi.org/10.1037/0022-3514.67.6.1049

Wytykowska, A., \& Gabińska, A. (2015). The effect of emotions, promotion vs prevention focus, and feedback on cognitive engagement. Polish Psychological Bulletin, 46(3), 350-361. https://doi.org/10.1515/ppb-2015-0042 
Zhang, S., Higgins, E. T., \& Chen, G. (2011). Managing others like you were managed: How prevention focus motivates copying interpersonal norms. Journal of Personality and Social Psychology, 100(4), 647-663. https://doi.org/10.1037/a0021750 